\title{
Comparative genomics of 84 Pectobacterium genomes reveals the variations related to a pathogenic lifestyle
}

\author{
Xiaoying $\mathrm{Li}^{1,2+}$, Yali Ma ${ }^{1,2+}$, Shuqing Liang ${ }^{3 \dagger}$, Yu Tian ${ }^{1,2}$, Sanjun Yin ${ }^{3}$, Sisi Xie ${ }^{3}$ and Hua Xie ${ }^{1,2^{*}}$
}

\begin{abstract}
Background: Pectobacterium spp. are necrotrophic bacterial plant pathogens of the family Pectobacteriaceae, responsible for a wide spectrum of diseases of important crops and ornamental plants including soft rot, blackleg, and stem wilt. $P$. carotovorum is a genetically heterogeneous species consisting of three valid subspecies, $P$. carotovorum subsp. brasiliense (Pcb), P. carotovorum subsp. carotovorum (Pcc), and P. carotovorum subsp. odoriferum (Pco).

Results: Thirty-two $P$. carotovorum strains had their whole genomes sequenced, including the first complete genome of $P c O$ and another circular genome of $P c b$, as well as the high-coverage genome sequences for 30 additional strains covering Pcc, Pcb, and Pco. In combination with 52 other publicly available genome sequences, the comparative genomics study of $P$. carotovorum and other four closely related species $P$. polaris, P. parmentieri, $P$. atrosepticum, and Candidatus P. maceratum was conducted focusing on CRISPR-Cas defense systems and pathogenicity determinants. Our analysis identified two CRISPR-Cas types (I-F and I-E) in Pectobacterium, as well as another I-C type in Dickeya that is not found in Pectobacterium. The core pathogenicity factors (e.g., plant cell wall-degrading enzymes) were highly conserved, whereas some factors (e.g., flagellin, siderophores, polysaccharides, protein secretion systems, and regulatory factors) were varied among these species and/or subspecies. Notably, a novel type of T6SS as well as the sorbitol metabolizing srl operon was identified to be specific to Pco in Pectobacterium.

Conclusions: This study not only advances the available knowledge about the genetic differentiation of individual subspecies of $P$. carotovorum, but also delineates the general genetic features of $P$. carotovorum by comparison with its four closely related species, thereby substantially enriching the extent of information now available for functional genomic investigations about Pectobacterium.
\end{abstract}

Keywords: Pectobacterium carotovorum, Comparative genomics, Genetic variation, Pathogenicity determinants, CRISPR-Cas

\section{Background}

Pectobacterium spp. are necrotrophic bacterial plant pathogens of the family Pectobacteriaceae, responsible for a wide spectrum of diseases (including soft rot, blackleg, and stem wilt) of important crops and ornamental plants worldwide [1]. At the time of writing, the genus has ten species ( $P$. carotovorum, $P$. wasabiae, $P$.

\footnotetext{
* Correspondence: xiehua@baafs.net.cn

${ }^{\dagger}$ Xiaoying Li, Yali Ma and Shuqing Liang contributed equally to this work. ${ }^{1}$ Beijing Agro-Biotechnology Research Center, Beijing Academy of Agriculture and Forestry Sciences, Beijing 100097, People's Republic of China

${ }^{2}$ Beijing Key Laboratory of Agricultural Genetic Resources and Biotechnology, Beijing 100097, People's Republic of China

Full list of author information is available at the end of the article
}

betavasculorum, $P$. cacticida, $P$. aroidearum, $P$. peruviense, $P$. atrosepticum, $P$. parmentieri, $P$. polaris, and Candidatus P. maceratum), of which $P$. carotovorum, $P$. atrosepticum, and $P$. parmentieri are three soft rot species that cause the most severe economic losses [2, 3]. In contrast to $P$. atrosepticum and $P$. parmentieri, the causal agent of potato rots, $P$. carotovorum exhibits a wide host range and high heterogeneity, and is further divided into three valid subspecies: $P$. carotovorum subsp. brasiliense $(P c b)$, P. carotovorum subsp. carotovorum (Pcc), P. carotovorum subsp. odoriferum (Pco), as well as a the newly proposed but as yet not validly published subspecies $P$. carotovorum subsp. actinidiae (Pca) [4-6]. In addition, some strains previously known as

(c) The Author(s). 2018 Open Access This article is distributed under the terms of the Creative Commons Attribution 4.0 International License (http://creativecommons.org/licenses/by/4.0/), which permits unrestricted use, distribution, and 
P. carotovorum has been separated from P. carotovorum recently and have been proposed as four new species: $P$. aroidearum, $P$. peruviense, $P$. polaris and Candidatus P. maceratum [7-10].

Pectobacterium spp. possess a great number of pathogenicity determinants that contribute to the ability to cause maceration-associated diseases [4]. Most of them are secreted and injected into host cells through one or a combination of the six known types of bacterial protein secretion systems: T1SS-T6SS [11]. Recent studies on CRISPR-Cas systems, which function as a type of microbial immune system, may also influence Pectobacterium pathogenicity [12]. We focus our studies on genome-level variation in known virulence factors such as Plant Cell Wall Degrading Enzymes (PCWDEs), secretion systems, flagellin, siderophores, and polysaccharides, as well as CRISPR-Cas systems, in genus Pectobacterium, and especially in the heterogeneous species $P$. carotovorum in order to improve our understanding of the genetic basis for pathogenicity in general.

Genome sequencing has provided new insights into the lifestyle of Pectobacterium. P. atrosepticum SCRI1043 (accession: BX950851) was the first soft rot pathogen sequenced in 2004 [11]. By July 2018, many genome data have been released successively of which 16 more Pectobacterium complete genome sequences have become publicly available: P. atrosepticum 21A (accession: CP009125) [13], P. atrosepticum JG10-08 (accession: CP007744), P. wasabiae CFBP3304 (accession: CP015750), P. aroidearum PC1 (accession: CP001657), P. parmentieri WPP163 (accession: CP001790), P. parmentieri SCC3193 (accession: CP003415) [14], P. parmentieri RNS08.42.1A (accession: CP015749) [3], P. carotovorum 3-2 (accession: CP024842) and PCC21 (accession: CP003776) [15], Candidatus P. maceratum SCC1 (accession: CP021894) [16], P. polaris NIBIO1392 (accession: CP017482) and NIBIO1006 (accession: CP017481) [9], Pcb SX309 (accession: CP020350) and BZA12 (accession: CP024780) [17], as well as two new sequenced genomes Pcb BC1 (CP009769), Pco BCS7 (accession: CP009678) in this study (Additional file 1: Table S1). Previous genomic studies have compared Pectobacterium with other genera [18-20], and we are aware of two studies that examined Pectobacterium species/subspecies differences among $P$. atrosepticum, $P c c$, and $P c b$ [21], and another comparing P. parmentieri and P. atrosepticum [22]. However, no reports have as yet compared the genomes of all of the subspecies of $P$. carotovorum.

The present study provides an important resource for functional genomics studies of the heterogeneous $P$. carotovorum. A total of $32 P$. carotovorum strains including 14 Pcb, 5 Pcc, and $13 P c o$ strains were collected from 3 economically important Chinese vegetables (Chinese cabbage, Bok choy, and celery) (Additional file 1: Table S1 and Additional file 2: Figure S1), and these were characterized by conducting full genome sequencing and extensive phenotypic analyses. In addition, we conducted comparative genomics based on the 32 newly sequenced sequences and 52 additional genomes from P. carotovorum, P. polaris, Candidatus $P$. maceratum, $P$. atrosepticum, and $P$. parmentieri available from GenBank with three complete Dickeya genomes (D. zeae Ech586, D. paradisiaca Ech703, and D. dadantii 3937) as the reference. Our results not only represent progress in the knowledge regarding the genetic differentiation of individual subspecies of $P$. carotovorum, but also delineate the general genetic features of $P$. carotovorum by comparison with that of other four closely related species in following aspects: phylogenetic analysis, general genomic properties, genome similarity, pan-genome, CRISPR-Cas systems, pathogenicity-related genes, and TNSSs. The results showed the $\mathrm{srl}$ operon/T6SSc is apparently specific to Pco subspecies, and we therefore further studied the distribution and the similarity of $\mathrm{srl}$ operon/T6SSc in the Pectobacterium genus and even in the Enterobacterales order by using an expanding genome data set. This study represents a major step towards filling in our understanding about genomic differentiation in pathogenicity-related genes and pathways of the economically important Pectobacterium soft rot pathogens.

\section{Result \\ Phenotypic and genomic features of 32 Chinese Pectobacterium carotovorum isolates}

Thirty-two Chinese soft rot isolates including $14 P c b, 5$ $P c c$, and 13 Pco strains were collected from 3 economically important Chinese vegetables (Chinese cabbage, bok choy, and celery) afflicted with bacterial soft rot disease (Additional file 2: Figure S1). All isolates tested caused soft rot lesions at the locations that were inoculated onto Chinese cabbage petioles (Additional file 1: Table S1). The extent of the decay caused by the $P c b$ strains (mean diameter of decay in Chinese cabbage $=3.29 \mathrm{~cm}$ ) was, on average, less substantial than the decay caused by the $P c c$ or Pco strains (mean diameter of decay $=3.62$ and $3.72 \mathrm{~cm}$, respectively); however, consider that the $P c b$ strains exhibited a very wide range of values $P c b$ $(0.24 \sim 4.06 \mathrm{~cm}$ ), $P c c$ (range $3.13 \sim 3.83 \mathrm{~cm}$ ), and $P c o$ (range $1.19 \sim 4.20 \mathrm{~cm}$ ). All tested strains were able to grow at $37^{\circ} \mathrm{C}$ and were positive for utilization of cellobiose, sucrose, $\alpha$-D-glucose, D-fructose, D-mannitol, and glycerol. They were unable to utilize propionic acid, L-alanine, D-maltose, D-serine, tween 40, and L-histidine. However, all tested Pco strains produced acid from D-sorbitol, palatinose, $\alpha$ methylglucoside, and utilized D-arabitol whereas the $P c c$ and $P c b$ strains could not (Additional file 1: Table S1-S2).

Subsequently, to examine their genomic variation that may underly phenotypic differences, we sequenced the 32 $P$. carotovorum isolates to an average depth of 100-fold 
coverage. The genomes of both Pcb $\mathrm{BC} 1$ and Pco BCS7 (Fig. 1a and b) consist of a single circular chromosome of $4,920,350 \mathrm{bp}$ and 4,933,575 bp, GC content of 51.84 and $51.75 \%, 4868$ and 3855 predicted CDS sequences, respectively, and both possessing 7 rRNA operons, and 77 tRNA genes. The sizes of the draft genomes for 30 additional $P$. carotovorum strains ranged from 4,710,507 to 5,144,582 bp with GC\% content ranging from 50.97 to $52.18 \%$ (Additional file 1: Table S3). No apparent autonomous plasmids were identified among these strains. We found that several genomic features that distinguish these Chinese Pco strains from others: the genomes of these Pco strains are the largest in size, have the lowest GC content, and have the least number of both tandem repeats and minisatellite DNA sequences compared with these Chinese $P c c$ and $P c b$ strains (Fig. 1c, d, and e).

\section{Comparative genomics analysis of Pectobacterium carotovorum and other closely related species}

To enrich the extent of information available for functional genomic investigations about $P$. carotovorum, we conducted comparative genomics analyses of individual subspecies of $P$. carotovorum and compared $P$. carotovorum genetic features with those of $P$. atrosepticum, $P$. parmentieri, P. polaris, Candidatus P. maceratum. A total of 84 Pectobacterium genomes including the 32 newly sequenced P. carotovorum genomes and the other available 52 Pectobacterium genomes downloaded from GenBank in July 2018 were used in this analysis (Additional file 1: Table S3).
Genomics features for distinguishing closely related species Generally, we found three major genomic features of these 84 Pectobacterium genomes seemed to have regularity with their classification of species and subspecies, including GC content and the numbers of both long terminal repeats and minisatellite DNA sequences (Additional file 1: Table S4). The differing GC content of closely related species has been considered as a useful means of discrimination for a long time [23]. At the subspecies level, Pco and Pca had lower GC content (51.27-51.70\%) than the other two subspecies ( $P c c$ and $P c b)$ (51.84-52.18\%; except Pcc Y16 (50.97\%) and $P c b$ CFIA1033 $(51.25 \%))$. At the species level, P. carotovorum had a similar GC content with $P$. polaris and Candidatus P. maceratum, which was higher than $P$. atrosepticum (50.87-51.15\%), whereas $P$. parmentieri (50.37-50.59\%) had the lowest GC content among these four species. Overall, these Pectobacterium spp. had a lower GC content (50.37-52.18\%) in comparison with the three Dickeya strains (53.63-56.29\%). There were also fewer long terminal repeats in $P$. carotovorum, $P$. polaris, and Candidatus $P$. maceratum (56122; except Pcc WPP14 (134), P $c b$ PBR $1692^{\mathrm{T}}$ (137), and Pca ICMP 19971 (126)) than in the other two species $P$. atrosepticum and $P$. parmentieri (125-157). For minisatellite DNA sequences, $P c a$ had the lowest number (84-88) among these subspecies and species, and the number of Pco (115-129; except NCPPB3841 (143)) was less than that of $P c c / P c b(131-172)$ at the subspecies level.
A

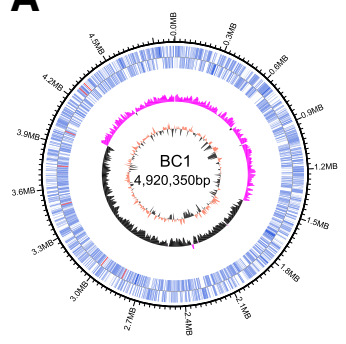

B

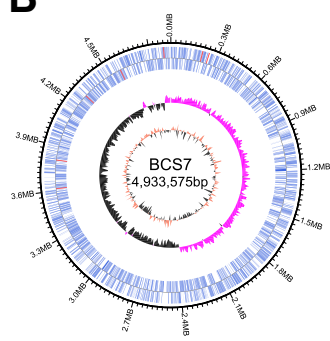

C

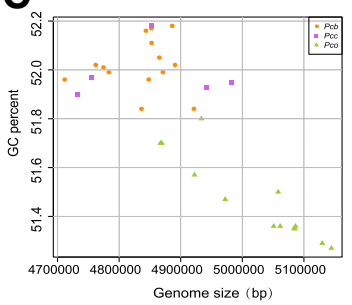

D

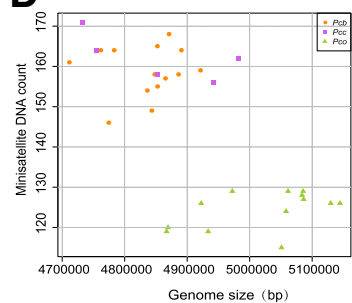

E

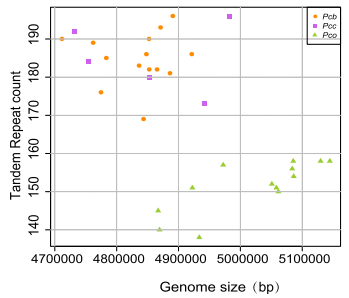

Fig. 1 Genomic features of the 32 newly sequenced Pectobacterium carotovorum strains. a Circular chromosome map of Pcb strain BC1. b Pco strain BCS7. The distribution of genes is shown on the two outer rings. The next circle indicates the GC-skew values (pink: the GC-skew values > the average; black: the GC-skew values < the average) and the central circle shows the GC content (salmon: the GC content> the average; black: the GC content < the average). Two-dimensional plots showing relationships between genome size and GC content (c), minisatellite DNA count (d), and TR count (e) for the 32 newly sequenced $P$. carotovorum strains. The salmon dots represent the 14 Pcb strains, the pink squares represent the 5 Pcc strains, and the light green triangles represent the 13 Pco strains 
In addition, $P$. parmentieri had fewer such sequences (94-100) than $P$. atrosepticum (124-141), which was far fewer than that in Candidatus P. maceratum or $P$. polaris (148-208) at the species level.

\section{Genome similarity of Pectobacterium carotovorum and other species as well as that of $P$. carotovorum subspecies} The topologies of maximum likelihood tree based on the huge SNP data from the 84 Pectobacterium genomes revealed that the four closely related species formed two major well-resolved groups (Fig. 2a). One group consisted of species P. carotovorum, P. polaris, and Candidatus P. maceratum, while the species $P$. parmentieri and $P$. atrosepticum formed the other group. Strains of $P$. carotovorum were further clustered into four distinct clades: clade 1, for $P c b$ strains and another one $P c c$ strain (ATCC 39048); clade 2, for Pcc strains; clade 3, for Pco strains; and clade 4, for Pca strains. Clade 1 was split further as follows: one subclade contained the type strain Pcb LMG $21371^{\mathrm{T}}$ as well as another five published $P c b$ strains from Brazil, New Zealand, and South Africa, and the other subclade harbored all the strains of the subspecies isolated from China, Russia, Canada, Korea as well as one $P c c$ strain from USA. This indicates that significant genetic diversity exists among different $P c b$ strains related to the origins, and the Pcc strain ATCC 39048 appear to be misnamed. It was also showed that a close relationship exists between $P c c$ and $P c o$, as they were gathered into a single large group that was separated from the $P c b$ strains. The Chinese Pco strains isolated from celery and Chinese cabbage were clustered with the type strain of Pco NCPPB $3839^{\mathrm{T}}$ and the other Pco strain isolated from chicory. In addition, two strains P. carotovorum 3-2 and Pcc Ecc71 were clustered with the other published Candidatus P. maceratum strains suggesting an erroneous classification.

To characterize genomic similarity within each of subspecies/species, identity scores (IS) (Fig. 2b) were calculated based on the detected 52,630 SNPs among these tested strains. At the species level, we found $P$. carotovorum was most similar to Candidatus P. maceratum $(90.28-93.49 \%)$ and least similar to $P$. atrosepticum (83.62-84.94\%) and P. parmentieri (83.28-85.76\%). Within subspecies carotovorum, $P c b$ was more closely related to $P c c$ than to Pco and Pca, since Pcb strains had the higher IS values with $P c c$ strains (91.25-93.39\%) than with Pco
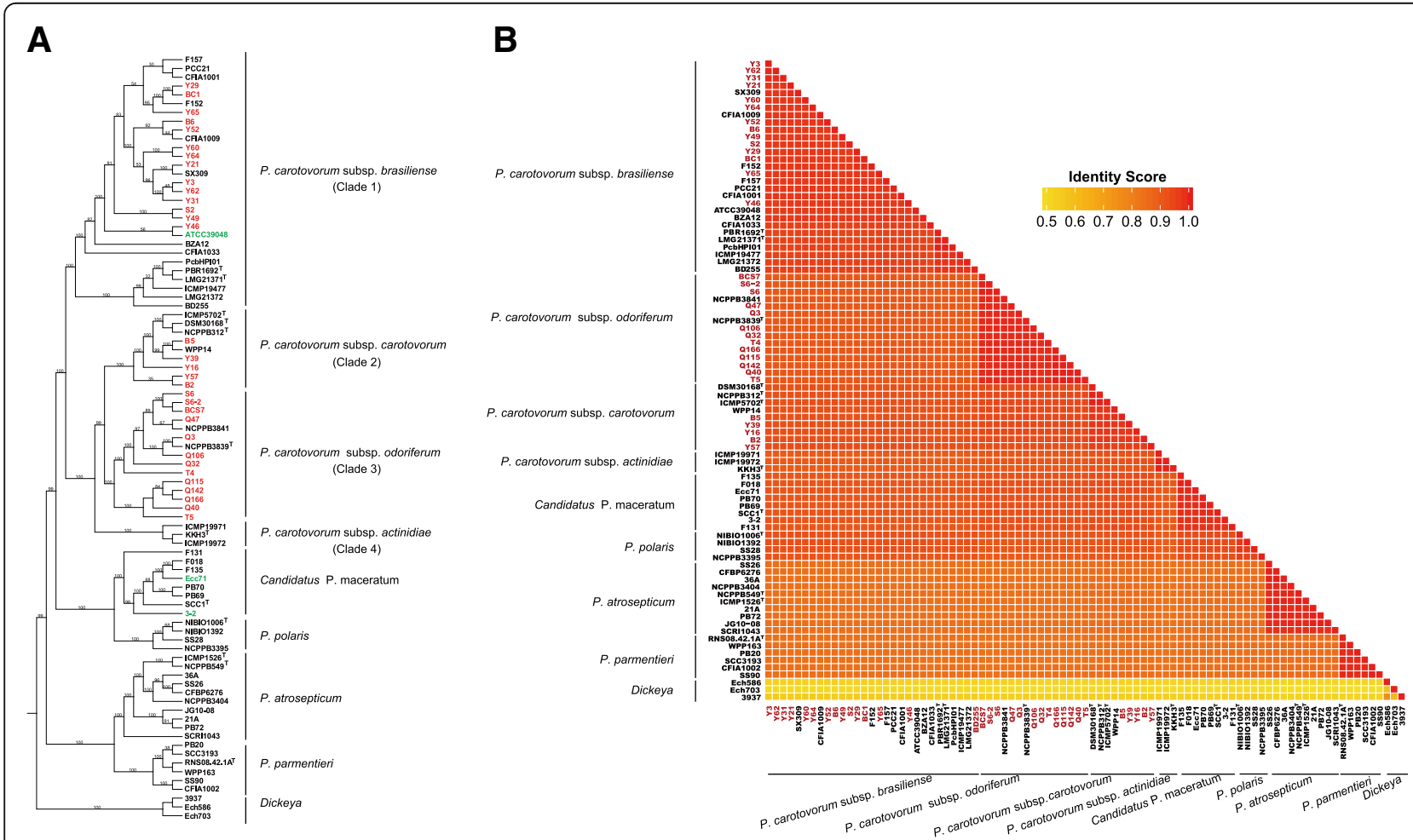

Fig. 2 Taxonomy and Identity Score analysis of the 84 Pectobacterium genomes. a Maximum likelihood tree based on SNP data for the 84 Pectobacterium genomes including 56 P. carotovorum (that is 9 Pcc, 29 Pcb, 15 Pco, and 3 Pca genomes), 6 P. parmentieri, 10 P. atrosepticum, 4 P. polaris, and 8 Candidatus P. maceratum genomes, with 3 Dickeya genomes (D. paradisiaca Ech 703, D. zeae Ech586, and D. dadantii 3937) as the outgroup. The tree was generated with 1000 bootstrap replicates. The strains with red letters are the newly sequenced Pectobacterium strains in this study and the three $P$. carotovorum strains with green letters are the apparently misnamed strains detailed in the text. b Heat map (color code in the ramp, above) of identity scores based on pairwise comparison of these 84 Pectobacterium genomes and 3 Dickeya genomes 
(89.65-90.63\%) and Pca strains (89.01-89.78\%). A close relationship was also observed between $P c c$ and Pco with higher similarity (92.79-93.79\%) than that of any other two subspecies. Pca strains also had high similarity with $P c c$ (91.31-91.80\%) and Pco (90.50-90.68\%) strains but had low similarity with $P c b$ (89.01-89.78\%). It is noteworthy that the similarity of three Korean $P c a$ strains $\left(\mathrm{KKH}^{\mathrm{T}}{ }^{\mathrm{T}}\right.$, ICMP 19971, and ICMP 19972) isolated from kiwi fruit $[6,24]$ were $100 \%$ amongst each other.

\section{Pan-genome analysis identified a srl operon specific to Pco} We performed a pan-genome analysis of Pectobacterium based on 36,634 putative protein-encoding sequences from the 84 Pectobacterium genomes sequences. The Pectobacterium pan-genome can be subdivided into three categories: (i) the core genome (that is, the set of genes present in the genome of at least one strain of each subgroup; not a gene that is shared by all strains), (ii) the accessory genome (the set of genes present in some but not all subgroups), and (iii) the unique genome (genes that are unique to a single subgroup) (Fig. 3). The core genome of Pectobacterium consists of 3171 (17.47\%) out of a total of 18,147 orthologous genes. OrthoMCL comparative analysis of shared genes revealed a higher number of genes commonly present in $P$. carotovorum-Candidatus P. maceratum (4762), and $P$. carotovorum- $P$. polaris (4657), than that in common between $P$. carotovorum-P. atrosepticum (4332), and $P$. carotovorum-P. parmentieri (4362), supporting our observations from the abovementioned IS analysis that showed a closer genetic relationship between $P$. carotovorum and Candidatus P. maceratum than with P. polaris, P. atrosepticum, or P. parmentieri (Fig. 3a). The core orthologous genes and subspecies-specific unique

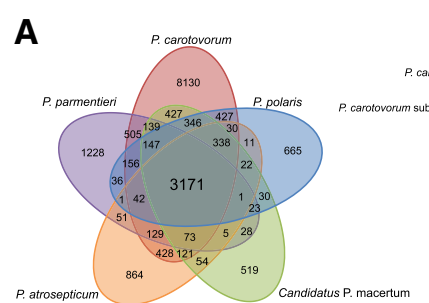

B
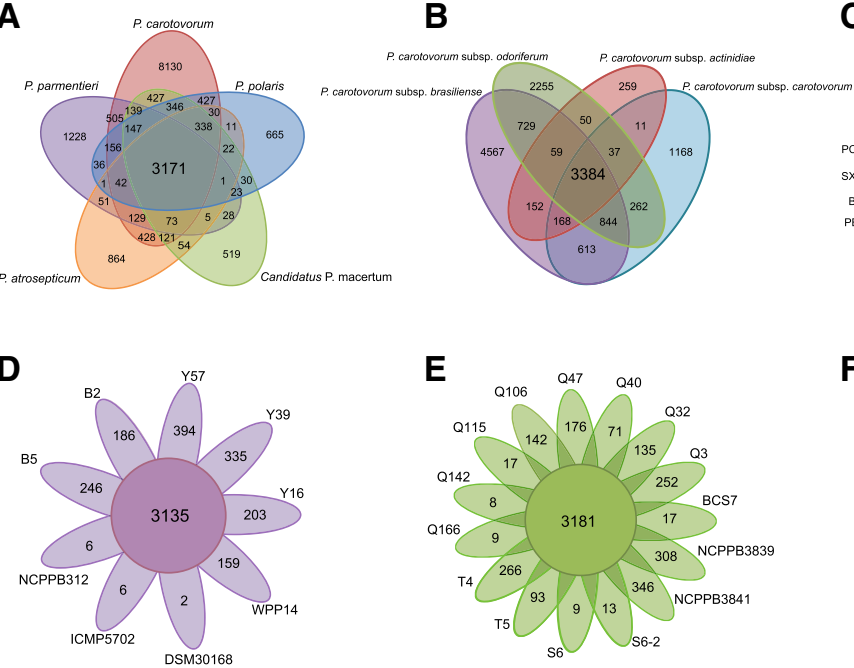

E

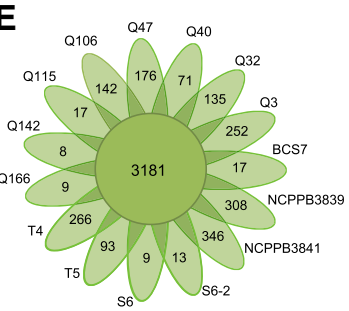

$\mathbf{F}$
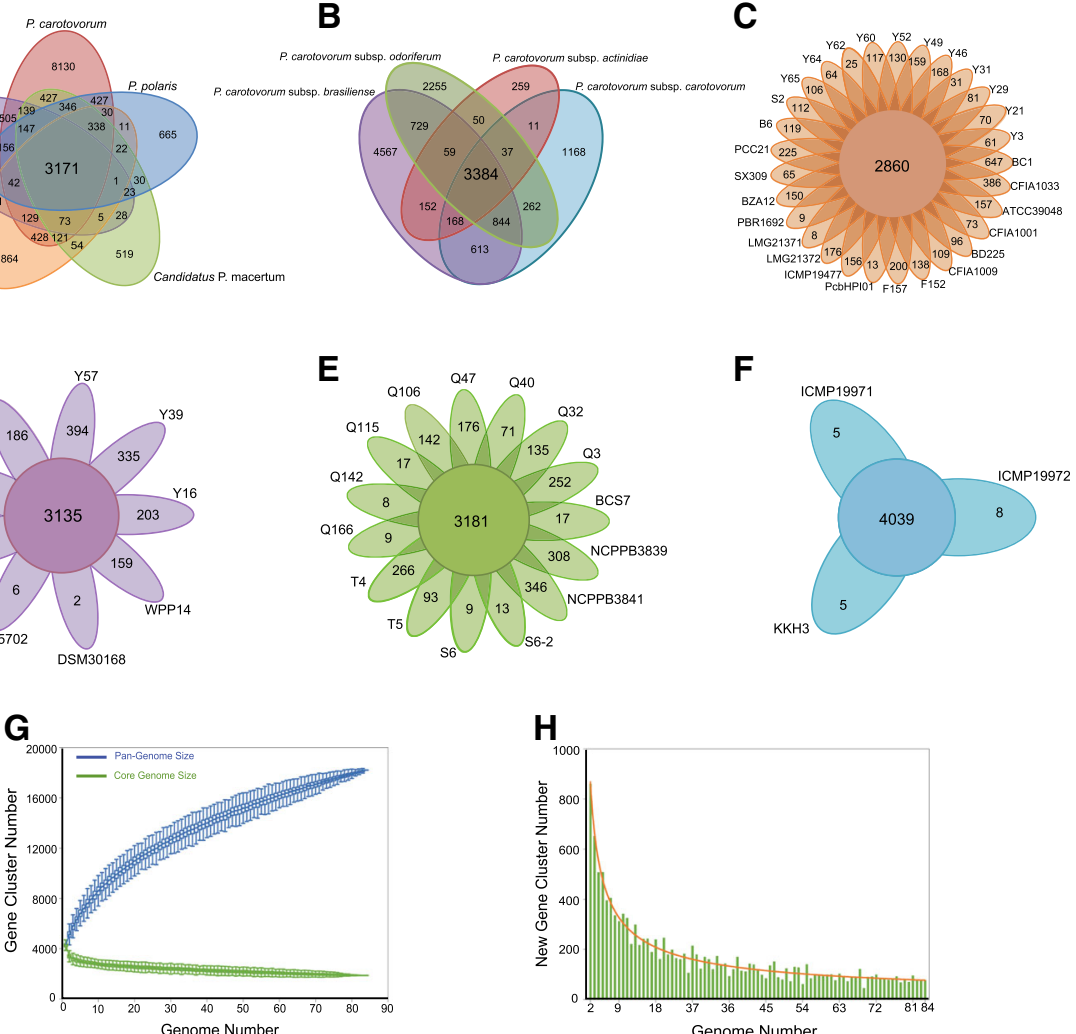

Fig. 3 Pan- and core-genome analyses of the 84 Pectobacterium genomes. The number of genes in the pan-genomes and core-genomes, and the unique genes among the genomes were classified using CD-HIT, with a threshold of $70 \%$ pairwise identity and a 0.6 length difference cut-off in amino acid sequences. a Venn diagram of Pectobacterium genus for 56 P. carotovorum, 4 P. polaris, 8 Candidatus P. maceratum, 10 P. atrosepticum, and 6 P. parmentieri genomes. $\mathbf{b}$ Venn diagram of $P$. carotovorum species for 29 Pcb, 9 Pcc, 15 Pco, and 3 Pca genomes. The core orthologous genes are not the genes that are shared by all of the tested genomes of a given group; rather these are the genes that were present in a minimum of one genome from each of the targeted subgroup. c-f Flower plots of each of four subspecies for 29 Pcb, 9 Pcc, 15 Pco, and 3 Pca genomes, respectively. The figures of 84 Pectobacterium genomes in $(\mathbf{g})$ and $(\mathbf{h})$ were produced using PanGP. $\mathbf{g}$ Plot in which the blue line shows the impact on the number clusters for the pan-genome resulting from the addition (simulated, randomized) of further genomes to the analysis, with the asymptotic value of $y=A x^{B}+C_{i}$ the green line shows how such addition influenced the number of core genes, with the asymptotic value of $y=A e^{B x}+C . \mathbf{h} H i s t o g r a m$ showing the increases in the number of new (unique) genes as the number of genomes was increased in our pan-genome analysis. The horizontal dashed line (orange) indicates the asymptotic value with the function of $y=A x^{B}$ 
genes within a given $P$. carotovorum species were examined in 9 Pcc, 15 Pco, $29 P c b$, and 3 Pca strains (Fig. 3b). A total of 14,558 homologs were identified. Of these, 3384 orthologs (23.24\%) were identified as the $P$. carotovorum core genome. A total of 5016 genes were shared between $P c o$ and $P c b$, while $P c c$ shared fewer orthologs with $P c b$ (5009) and $P c o$ (4527) and Pca shared few orthologs with the other three subspecies: $P c b$ (3763), Pcc (3600), and $P c o$ (3530), highlighting the relatively higher extent of genetic differentiation of Pca compared with the other three subspecies. The differentiation was also presented between different strains within the same subspecies in P. carotovorum (Fig. 3c, d, e, and f).

Additionally, the size of the pan-genome and its increase in size upon addition of new strains can be used to predict the future rate of discovering novel genes in a species (Fig. $3 g$ and $\mathrm{h}$ ): the pan-genome curve fit a Heaps' law pattern (exponent $>0$ ) and was considered "open", which is typical of species colonizing multiple environments and having several means of exchanging genetic material [25]. This analysis highlights the large genomic diversity of Pectobacterium species and suggests that these species can very likely adapt to a wide variety of hosts. The COG (Clustering of orthologous groups) analysis of unique genes of $P$. atrosepticum, $P$. parmentieri, $P$. polaris, Candidatus P. maceratum, as well as the subspecies of $P$. carotovorum showed that the srl operon (BCS7_10600-BCS7_10625) that is known to facilitate sorbitol utilization [26] was found only in the Pco strains, suggesting that this $s r l$ operon may be specific to Pco subspecies. The specific distribution of this operon in Pectobacterium genus were future confirmed in a subsequently expanded genome analysis using all of the available genomes from GenBank. These findings highlight substantial divergence among Pectobacterium species and subspecies. Seeking to better understand the genetic basis of pathogenicity and host specificity, our next analyses specifically compared the similarity and divergence of the genes encoding CRISPR-Cas, virulence determinants, regulatory mechanism, and protein secretion systems.

\section{The occurrence of I-E type CRISPR-Cas systems in Pectobacterium}

We investigated the CRISPR-Cas bacterial defense systems looking for genome-level variations that may influence the pathogenicity of Pectobacterium [12]. To date, surveys of the CRISPR-Cas systems in Pectobacterium have only been performed for P. atrosepticum SCRI1043, in which a single I-F type system was identified [27]. In the present study, we identified I-F, I-E type systems among these available Pectobacterium genomes, with seven exceptions among the Pectobacterium strains (Pcb LMG 21372, ICMP 19477, CFIA1001, and Y46; Pco NCPPB3841,
NCPPB $3839^{\mathrm{T}}$, and Q3; P. polaris NIBIO1006 ${ }^{\mathrm{T}}$ ) that lacked any CRISPR-Cas system (Table 1). The distribution of these systems varied among Pectobacterium species and subspecies: most of the P. carotovorum, P. polaris, and Candidatus P. maceratum strains contained I-F or I-E systems, and some strains carried both types (28\% Pcb, 33\% Pcc, $13 \%$ Pco, 25\% P. polaris, and 50\% Candidatus P. maceratum); all of the tested Pca and P. atrosepticum strains have a single I-F type, while both $P$. parmentieri strains have both the I-F and I-E types (Table 1). Moreover, our analysis identified CRISPR-Cas types (I-F, I-E and I-C) in reference Dickeya genomes, which have not been reported previously, as well as a I-C type in D. dadantii 3937 that is not found in Pectobacterium. The CRISPR-Cas systems from complete genomes of 84 Pectobacterium genomes, as well as three Dickeya genomes, are shown in Fig. 4, and the CRISPR-Cas systems of all the tested genomes in this study are shown in Additional file 1: Table S5.

\section{Inconsistently distributed pathogenicity-related genes among Pectobacterium species and subspecies}

To obtain a more comprehensive view of the pathogenicity factors present among Pectobacterium spp., we mined the 84 Pectobacterium genomes to identify previously known pathogenicity determinants, including PCWDEs, polysaccharides, iron uptake proteins, pili, and regulatory systems. Many pathogenicity determinants (e.g., most PCWDEs, enzymes for synthesis of enterobacterial common antigen (ECA) and the 3-hydroxy-2-butanone pathway) (Additional file 1: Table S6 and Table S6-1) are commonly existed among strains due to their importance in ensuring disease development caused by Pectobacterium spp. However, we also found inconsistent distributions of several single genes or large DNA fragments/clusters that possibly contribute to pathogenicity in variable or context-dependent scenarios for several species and subspecies (Table 2). All of the $P$. carotovorum, $P$. polaris, and Candidatus P. maceratum strains, but none of $P$. atrosepticum, and $P$. parmentieri strains, had the gene fhaB (BASYS01817) whose homolog encodes the filamentous hemagglutinin that influences virulence by human pathogens [28]. No regular distribution was observed on the two clusters (waa and $r f b$ ) encoding lipopolysaccharide (LPS) biosynthesis proteins present in $P$. carotovorum strains. The variation of LPS-related gene composition within the strains in this study likely reflects adaptations by Pectobacterium in the LPS-mediated plant-host interactions at the cell surface [22]. Notably, all of the $P$. parmentieri strains examined in this study lacked three PCWDEs genes (pel, pelA, and $p g l$ ) and a transport system for a ferric citrate cluster (fecABCDE), while possessing unique genes for siderophore biosynthesis and iron transport such as the genes iucABCD [29].

At the subspecies level, Pco exhibited distinctly different gene composition when compared with other closely 
Table 1 The identified types of CRISPR-Cas system in the 84 Pectobacterium genomes

\begin{tabular}{|c|c|c|c|c|}
\hline \multirow[t]{2}{*}{ Species/Subspecies } & \multirow[t]{2}{*}{ Strain } & \multicolumn{3}{|c|}{ Number of each CRISPR-Cas system type } \\
\hline & & $\mathrm{I}-\mathrm{F}$ & I-E & $\mathrm{I}-\mathrm{C}$ \\
\hline \multirow[t]{3}{*}{ P. carotovorum subsp. brasiliense } & $\begin{array}{l}\text { PBR1692 }^{\top}=L M G 21371^{\top}, \text { BC1, B6, Y3, Y29, Y31, Y60, Y62, } \\
\text { Y64, CFIA1009, CFIA1033, PcbHPI01, BD255, ATCC } \\
\text { 39048, F157, F152 }\end{array}$ & 1 & 0 & 0 \\
\hline & PCC21, SX309, BZA12, Y21, Y49, Y52, Y65, S2 & 1 & 1 & 0 \\
\hline & LMG 21372, ICMP 19477, CFIA1001, Y46 & 0 & 0 & 0 \\
\hline \multirow[t]{3}{*}{ P. carotovorum subsp. carotovorum } & Y57 & 1 & 0 & 0 \\
\hline & DSM $30168^{\top}=$ NCPPB $312^{\top}=$ ICMP 5702 ${ }^{\top}$, B2, Y16 & 0 & 1 & 0 \\
\hline & B5, Y39, WPP14 & 1 & 1 & 0 \\
\hline \multirow[t]{4}{*}{ P. carotovorum subsp. odoriferum } & Q40, Q106, Q115, Q142, Q166, T4, T5 & 1 & 0 & 0 \\
\hline & BCS7, S6, S6-2, & 0 & 1 & 0 \\
\hline & Q32, Q47 & 1 & 1 & 0 \\
\hline & NCPPB $3839^{\top}$, NCPPB3841, Q3 & 0 & 0 & 0 \\
\hline P. carotovorum subsp. actinidiae & ICMP 19972, ICMP 19971, KKH3 ${ }^{\top}$ & 1 & 0 & 0 \\
\hline \multirow[t]{3}{*}{ Candidatus P. maceratum } & F018, F135, Ecc71 & 1 & 0 & 0 \\
\hline & $3-2$ & 0 & 1 & 0 \\
\hline & SCC1 $^{\top}$, F131, PB69, PB70 & 1 & 1 & 0 \\
\hline \multirow[t]{3}{*}{ P. polaris } & NCPPB 3395, SS28 & 0 & 1 & 0 \\
\hline & NIBIO1392 & 1 & 1 & 0 \\
\hline & NIBIO1006 ${ }^{\top}$ & 0 & 0 & 0 \\
\hline P. atrosepticum & $\begin{array}{l}\text { ICMP } 1526^{\top}=\text { NCPPB } 549^{\top}, \mathbf{3 6 A}, \mathbf{2 1 A} \text {, CFBP 6276, } \\
\text { NCPPB 3404, PB72, SS26, JG10-08, SCRI1043 }\end{array}$ & 1 & 0 & 0 \\
\hline P. parmentieri & RNS08.42.1A', SCC3193, WPP163, SS90, CFIA1002, PB20 & 1 & 1 & 0 \\
\hline D. dadantii & 3937 & 1 & 0 & 1 \\
\hline D. zeae & Ech586 & 1 & 1 & 0 \\
\hline D. paradisiaca & Ech703 & 1 & 1 & 0 \\
\hline
\end{tabular}

The strains with bold black font are strains with completely sequenced genomes, whose CRISPR-Cas systems architectures are shown in Fig. 4, and the CRISPR-Cas systems of all strains in this study are shown in Additional file 1: Table S5

subspecies $P c a, P c b$ and Pcc. All Pco strains have the large DNA fragment (BCS7_01135-BCS7_01230) that contains several genes (pli, cpa, tad, and etc.) known to be involved in pili biosynthesis; this fragment is not present in other $P$. carotovorum strains with the exception of the $P c b$ strain BZA12. The nosA gene which encoded the out-membrane protein involved in the transport of metals [30] was absent in $P c b$ and $P c c$ while was present in Pco and Pca. Similarly, the Pco strains lack several genes carried by other subspecies strains, such as the major part of the pyelonephritis-associated pili (pap) operon (papACD; BASYS00091-BASYS00089), which are involved in the formation of adhesive pili in pathogenic bacteria [31,32]. We also identified intermittent distribution of AepA exoenzyme regulatory protein among $P c c$ and $P c b$ strains, but not at all in $P c o$ and $P c a$ strains. The AepA protein may enable Pectobacterium to use atypical nitrogen sources, which are secreted by the host plant or microorganisms that colonize the host plant's rhizosphere [33]. Many other genetic variations of pathogenicity related genes among these soft rot pectobacteria were shown in Table 2 and Additional file 1: Table S6. The findings of these unique genes confer to different species/subspecies provided a foundation for further work in elucidation of the genetic basis for pathogenicity in Pectobacterium.

\section{Three T1SS protein secretion systems (has, prt, and lap) in Pectobacterium}

The five protein secretion system types (T1SS-T4SS, and T6SS) found in 84 Pectobacterium genomes. Although all six secretion systems were reported to be present in Pectobacterium $[11,21]$ by Bell et al. and Glasner et al., improvements to annotation algorithms have resulted in the exclusion of T5SS in our study. The TNSSs of 3 Dickeya genomes used as the out-group also exhibited high diversity and were also discussed below.

Three T1SSs (T1SSa, T1SSb, and T1SSc) were identified among the Pectobacterium strains (Fig. 5a and Additional file 1: Table S7). While T1SSb (prtDEF) and 


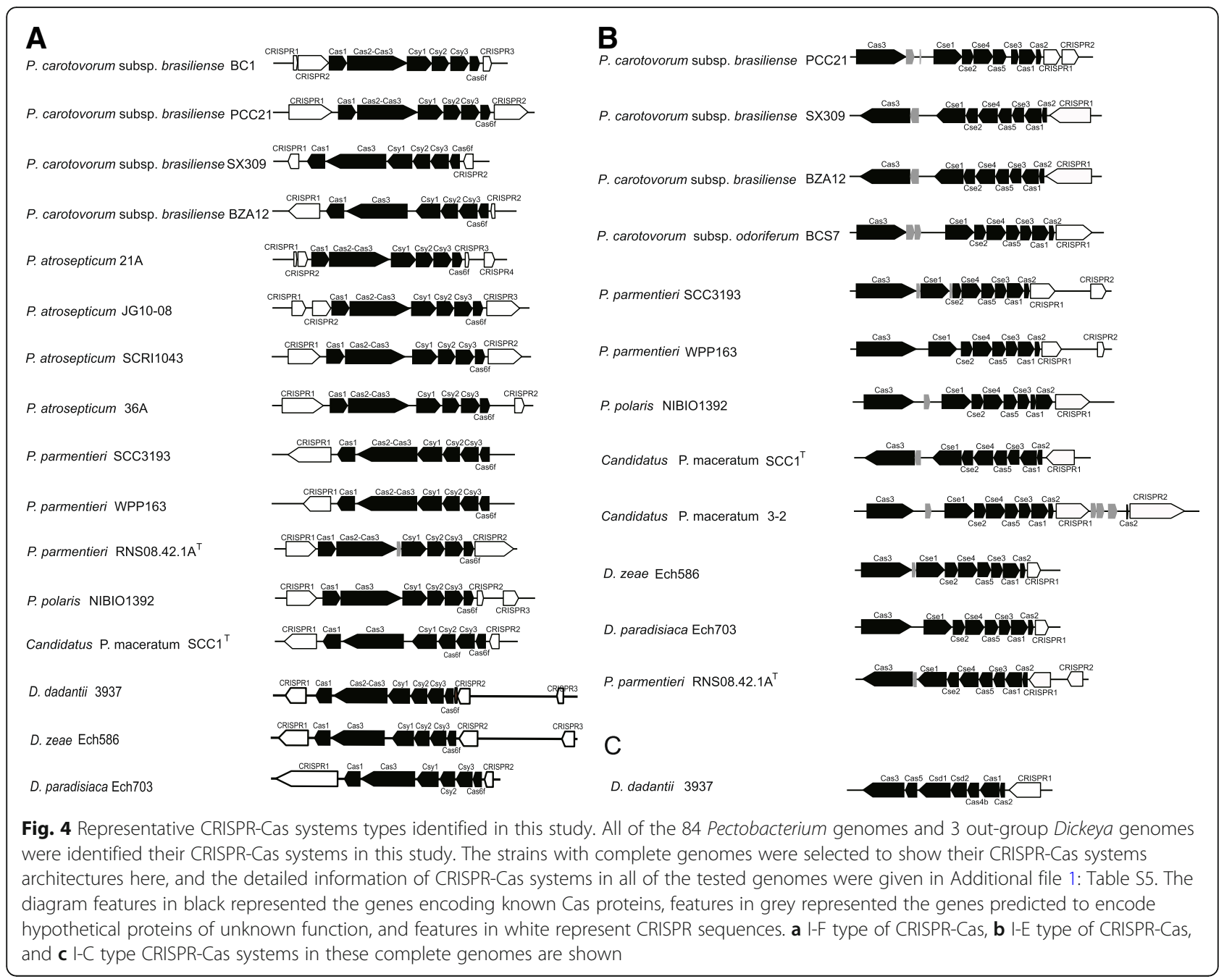

T1SSc (lapBCE) were present in all of the Pectobacterium strains in this study, T1SSa (hasDEF) was lost in $P$. parmentieri. Only T1SSb was found in the Dickeya strains we examined, of which D. paradisiaca Ech703 lacked the T1SSs. T1SSs secrete proteases and translocate molecules such as ions and toxins from the cytoplasm to the extracellular space [34], serving a range of functions. T1SSa of Serratia marcescens, for example, can translocate ions and secrete hemophores such as an extracellular heme-binding protein (HasA) to facilitate iron acquisition [35]. In Pectobacterium, T1SSb has been shown to secrete several Prt metalloproteases that are involved in attacking plant cell walls [36], and the repeats-in-toxin (RTX) protease PrtW mutants of $P$. parmentieri SCC3193 have altered virulence, suggesting a role for T1SSb in plant pathogenesis [37].

\section{Species- and subspecies-specific features of T2SS}

In contrast to the various T1SSs in Pectobacterium, only one T2SS cluster (T2SSa) was found among the
Pectobacterium strains (Fig. 5b). However, the P. parmentieri strains and three Dickeya strains carried a variant T2SSa (Gsp) lacking gspN compared with that of other Pectobacterium strains. GspN is predicted to have an amino-terminal transmembrane helix, but its function has not yet been determined [38]. We also found that the length of gsp $G$ was $441 \mathrm{nt}$ in all of $P$. atrosepticum and P. parmentieri strains and $456 \mathrm{nt}$ in Candidatus P. maceratum, but found that this varied among the $P$. carotovorum strains. Moreover, the intergenic sequence length between $g s p N$ and $g s p O$ was 89 nt in all Pco and Pcc strains, but was 108 nt in all $P c b$ strains and $88 \mathrm{nt}$ in all Pca strains. This intergenic length was not only specific to each subspecies, but was also specific at the species level, as the intergenic sequence length between $g s p N$ and $g s p O$ was $63 \mathrm{nt}, 123$ nt, and $89 \mathrm{nt}$, respectively, for each of the P. parmentieri, P. atrosepticum, and Candidatus P. maceratum strains. The extra T2SSb (Stt) presented in $D$. dadantii 3937 was not present in any tested Pectobacterium 


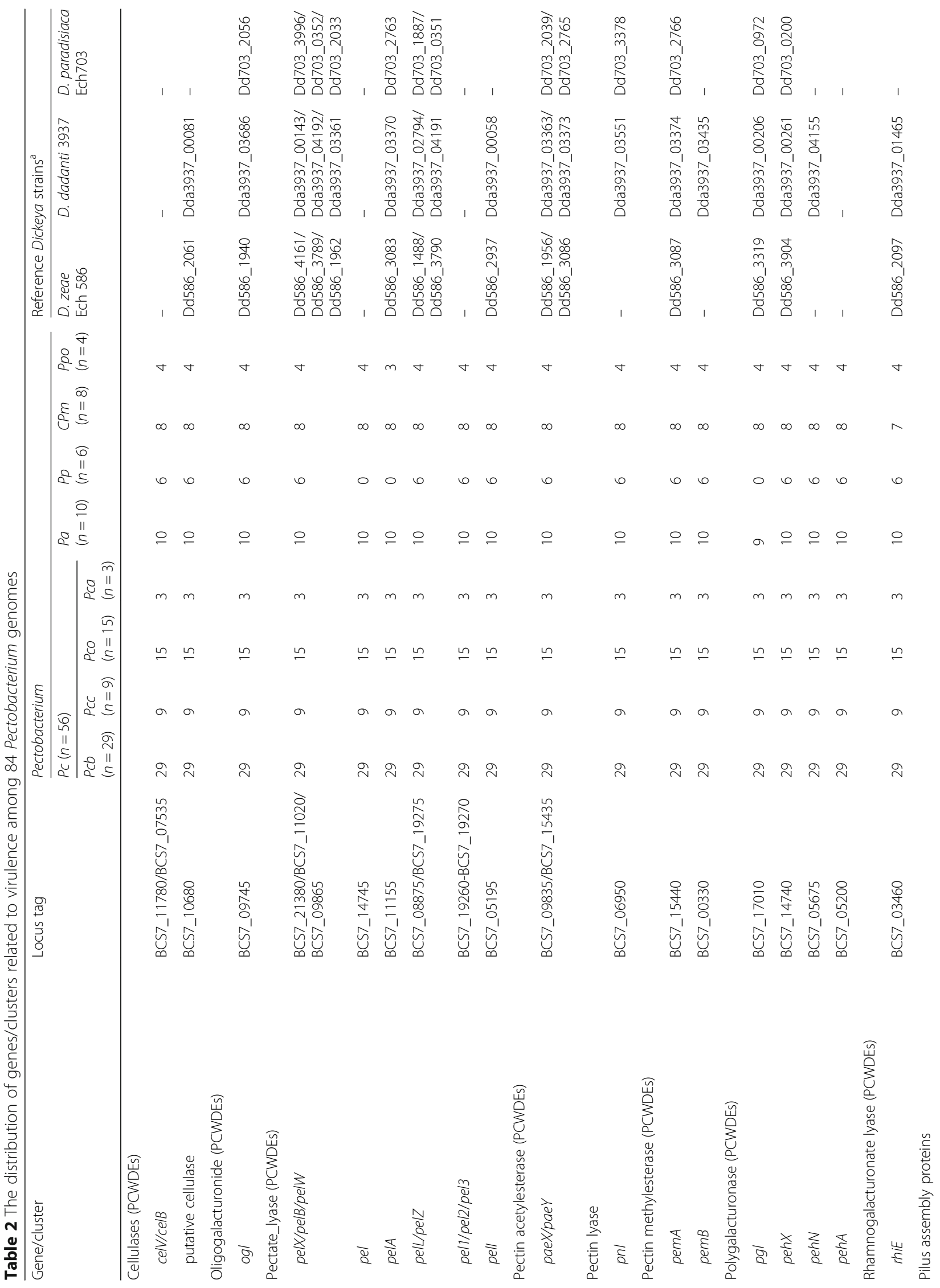


Li et al. BMC Genomics

(2018) 19:889

Page 10 of 22

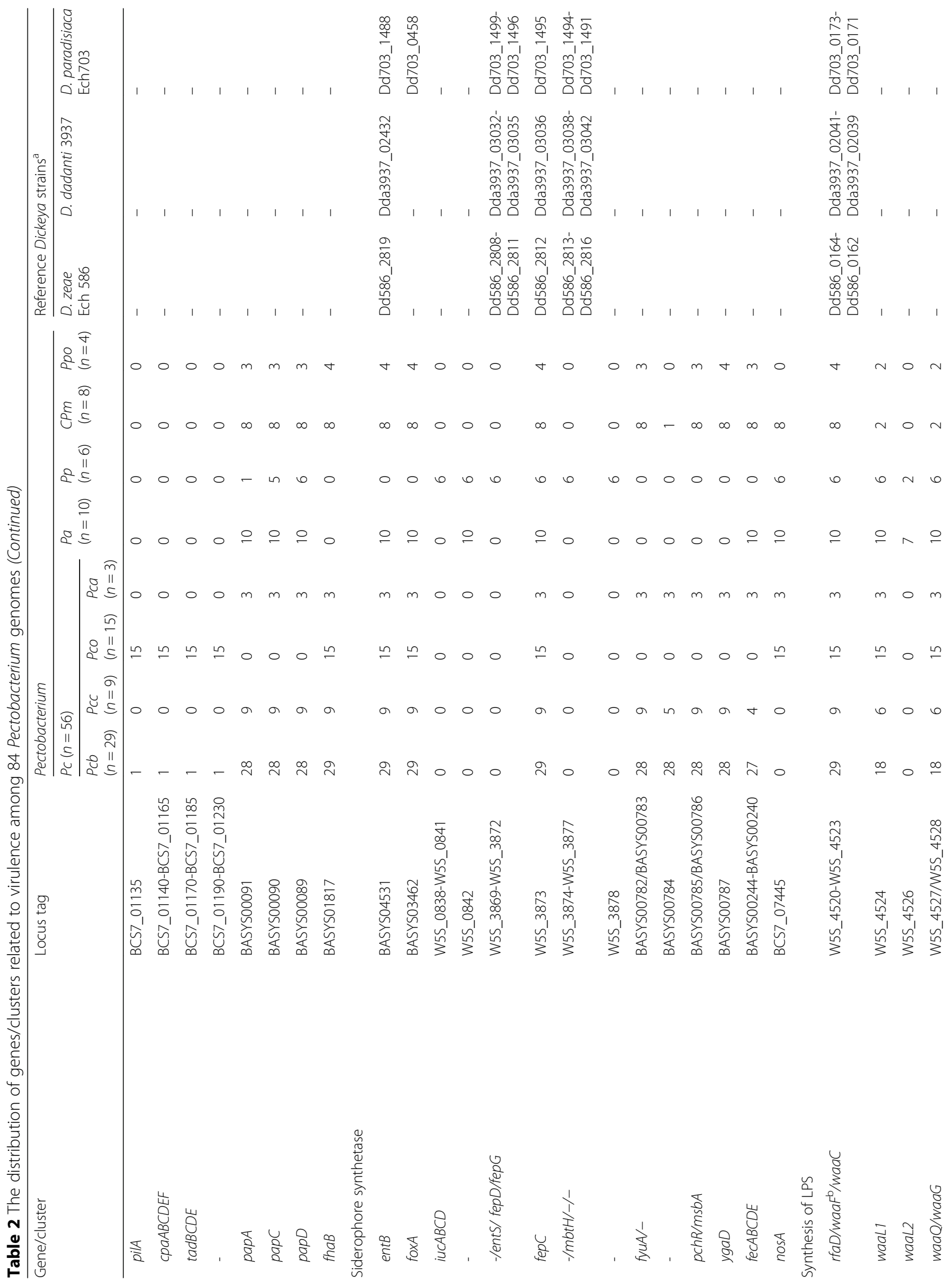




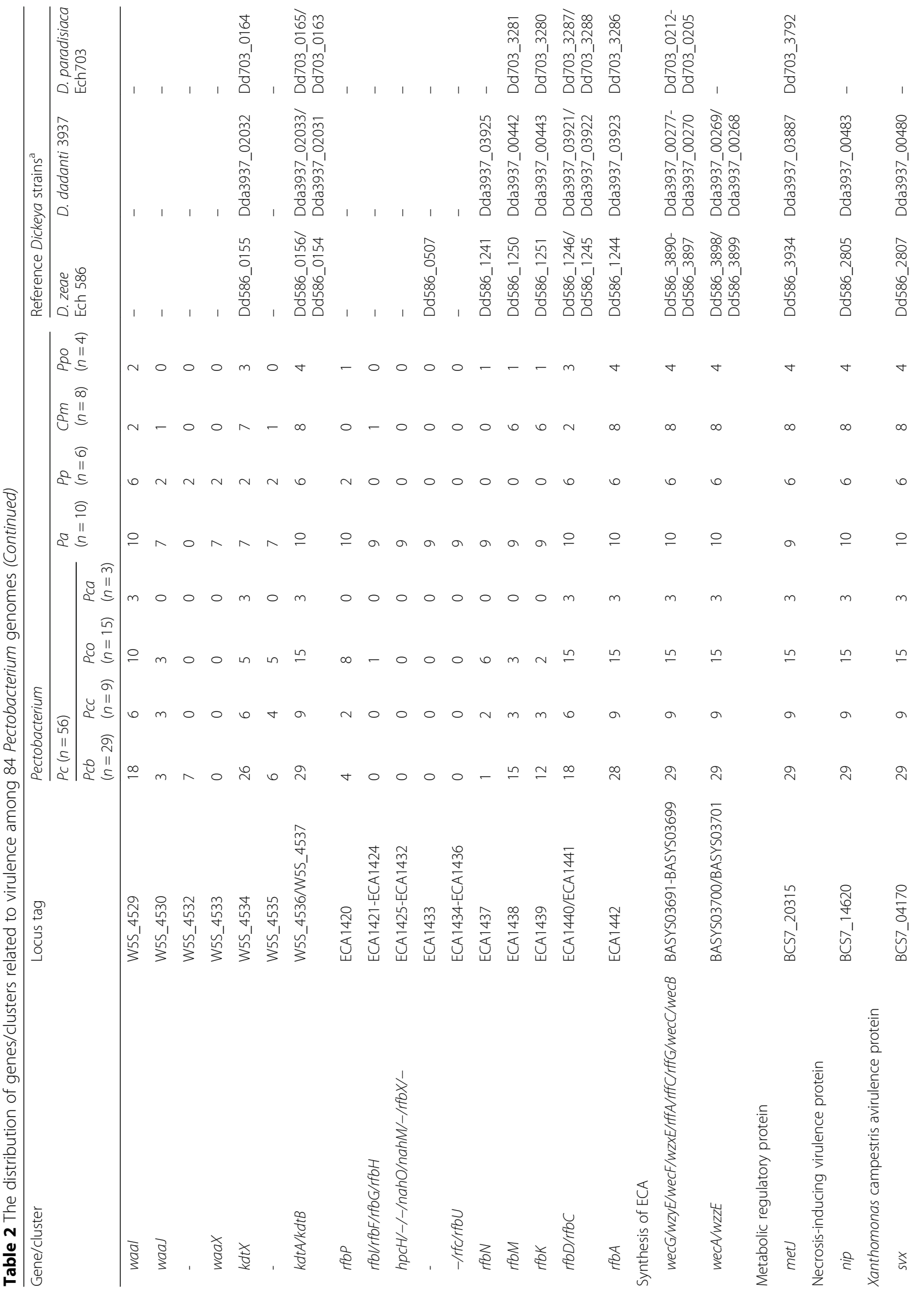


Li et al. BMC Genomics $\quad$ (2018) 19:889

Page 12 of 22

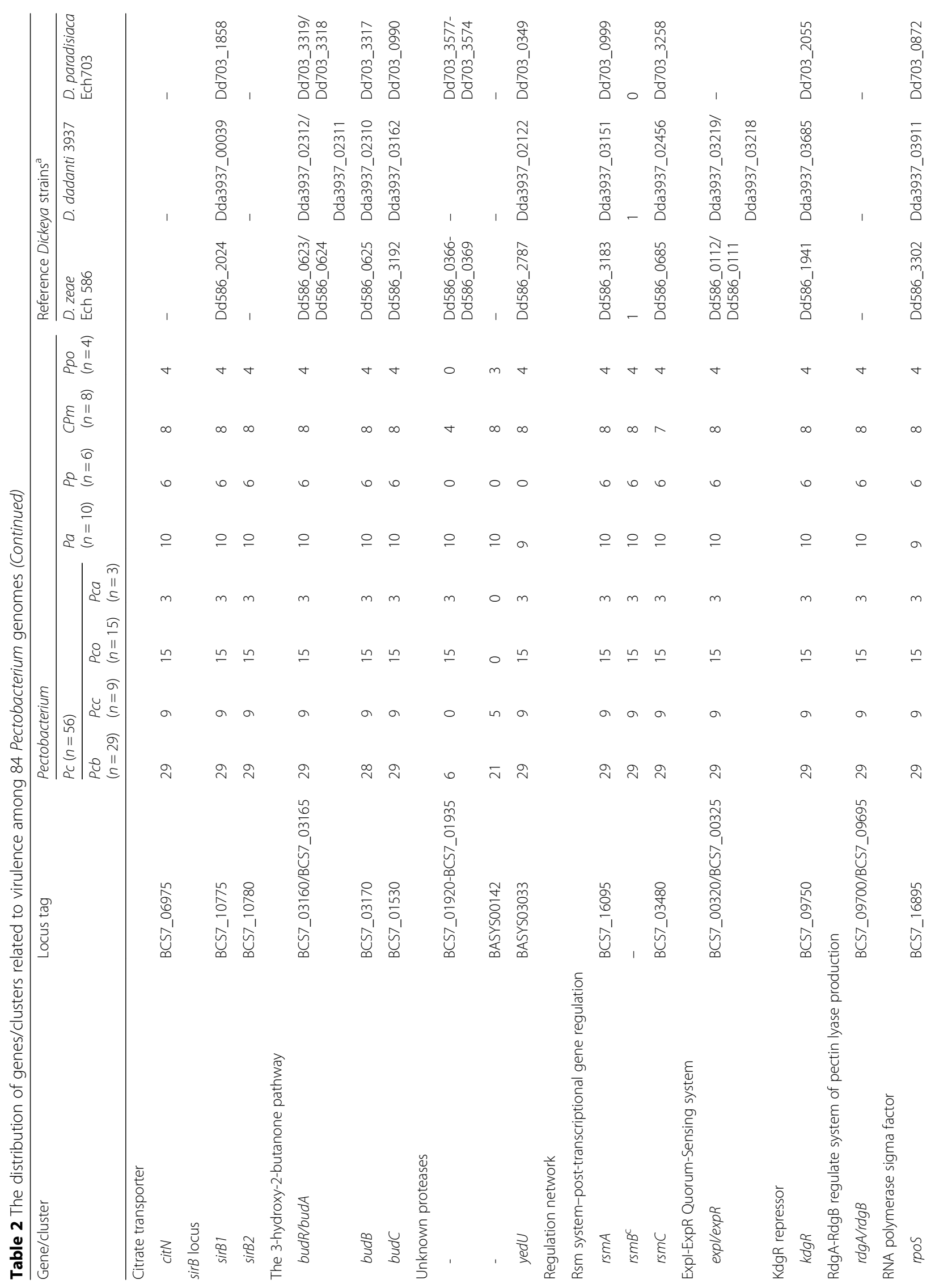




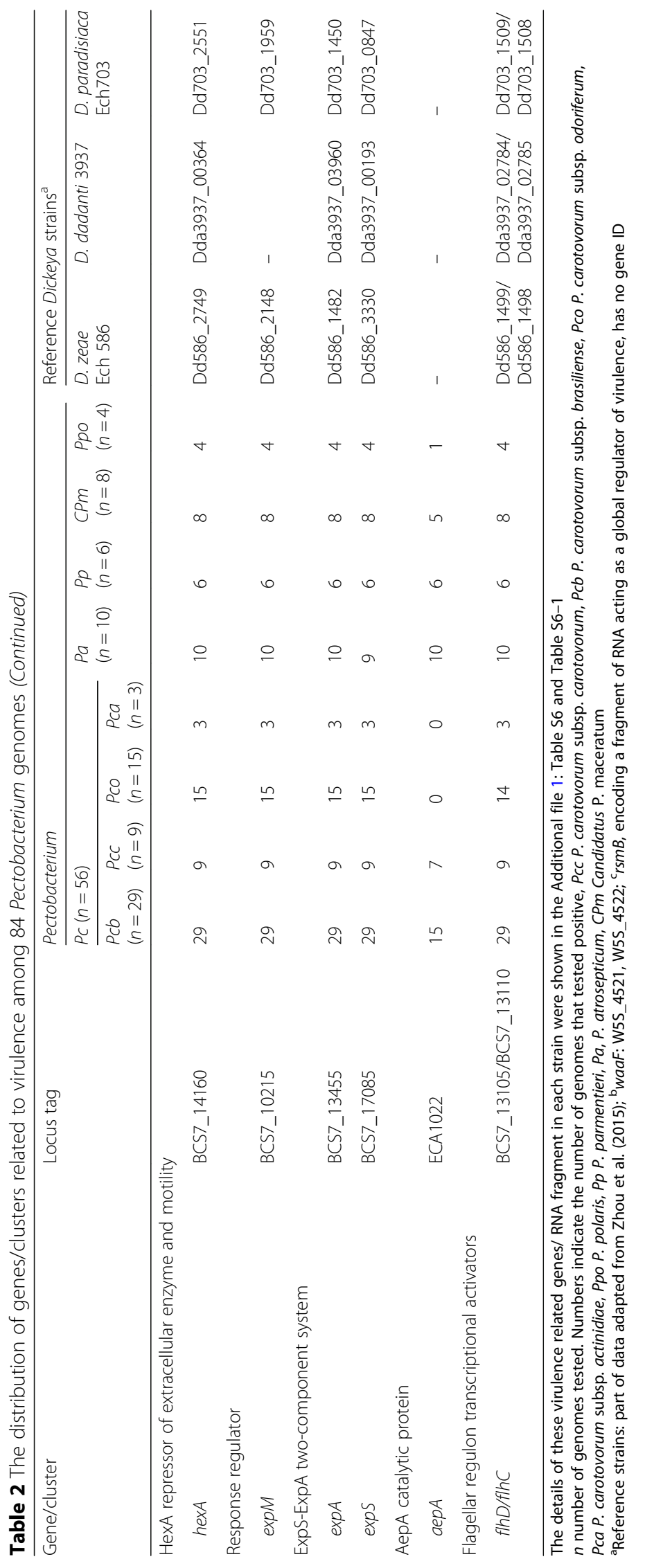




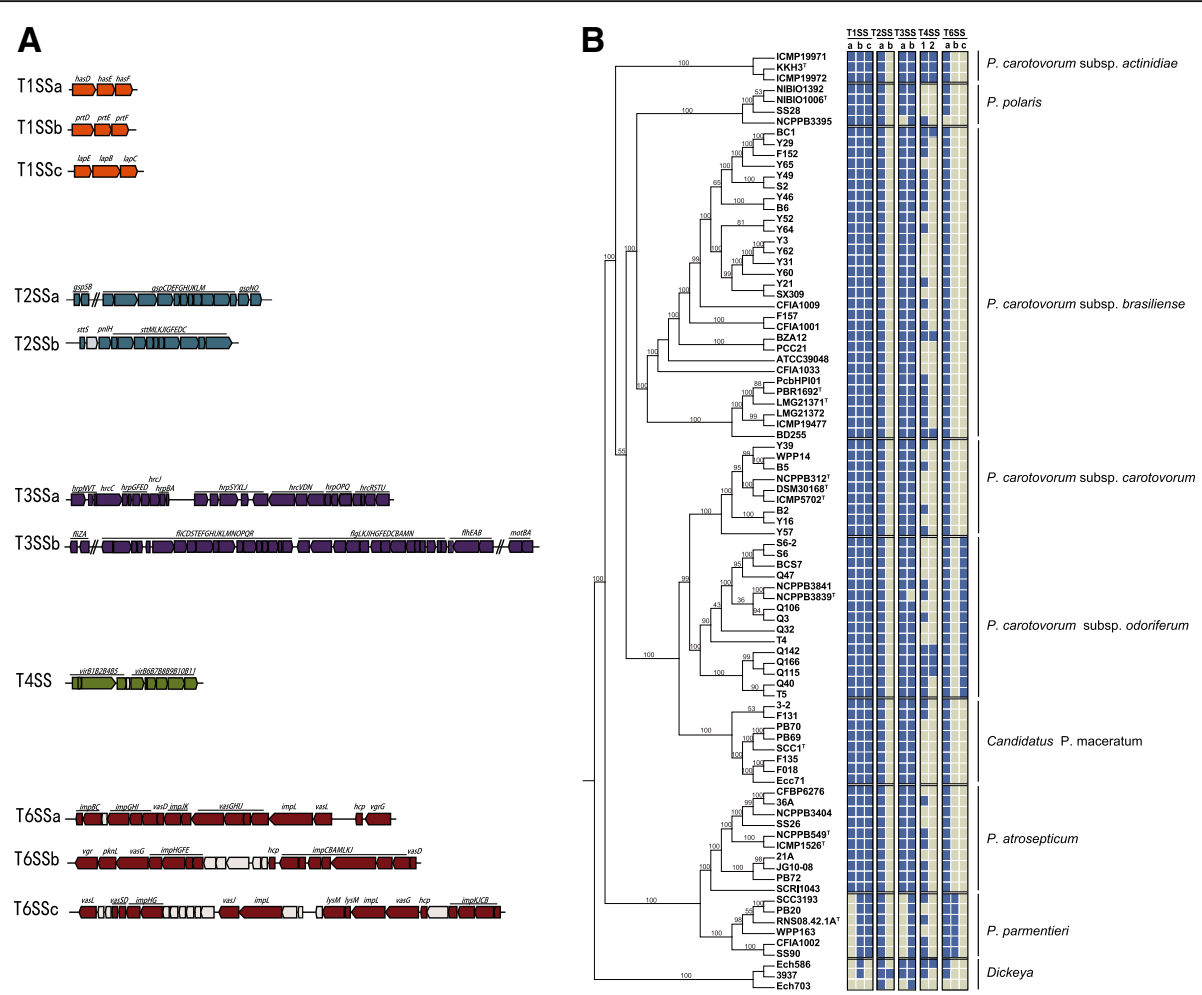

Fig. 5 Gene clusters for TNSSs identified in the 84 Pectobacterium genomes. a Physical maps of Type I, II, III, IV, and VI secretion systems. Red colored arrows indicate known genes involved in the protein secretion systems. b Map showing the presence (blue feature) or absence (grey feature) of each type of secretion systems gene cluster, with a maximum likelihood phylogenetic tree based on the core genes of all the tested strains. a-c represents different subtypes of a given secretion system gene cluster; $1-2$ represent the number of the copies of Type IV secretion system gene clusters

strains or in the other two Dickeya strains. T2SSb is known to encode a pectate lyase PnlH at the outer face of the outer membrane [39]. T2SS is extremely important for soft rot pathogens because it is responsible for the secretion of the major determinants of pathogenicity for this disease: the pectinases and cellulases that contribute to the characteristic rotting symptoms. It also secretes other factors related to pathogenicity, such as Svx and iron uptake proteins [40]. The inactivation of this secretory system renders Pectobacterium spp. avirulent [41].

\section{T3SSa is absent in Pectobacterium parmentieri but found in other species}

T3SSs include the virulence-associated Type III (T3SSa) and the flagellar secretion system (T3SSb). T3SSa clusters were absent from $P$. parmentieri, but were present in all of the strains of $P$. carotovorum, Candidatus $P$. maceratum, and P. atrosepticum (Fig. $5 \mathrm{~b}$ and Additional file 1: Table S7). These results were in agreement with previous studies that failed to detect T3SSa or any of its surrounding genes in P. parmentieri SCC3193, a strain known to be virulent [22]. It is likely that T3SSa is not strictly required for the virulence and survival of $P$. parmentieri, although this cluster has been reported to contribute modestly to the virulence of $P$. atrosepticum and $P$. carotovorum [42]. A previous study reported some naturally T3SS-deficient $P$. carotovorum strains [43]; however, one $P$. polaris strain NCPPB 3395 from the Netherlands, previously assigned to Pcc [9], were identified as a T3SSa-deficient strain in the present study. T3SSa was present in D. zeae Ech586 and D. dadantii 3937, but this was lost in the $D$. paradisiaca Ech703. Whereas T3SSa had a species-specific distribution, T3SSb (BASYS02177-BASYS02229), which encodes important flagella components [44], was highly conserved among these Pectobacterium spp. and three Dickeya genomes except one Pco strain (NCPPB $3839^{\mathrm{T}}$ ).

\section{Irregular distribution of T4SS in Pectobacterium}

T4SS is a versatile secretion system that can function in bacterial conjugation, DNA uptake/release, and cargo translocation. Several of the proteins encoded by the T4SS form three major sub-complexes: a cytoplasmic-inner membrane complex (VirB4 and VirB11), a core complex (VirB7, VirB9, and Vir10), and an extracellular pilus-associated complex (VirB2 and VirB5) (Fig. 5a). P. atrosepticum SCRI1043 VirB4 mutants are reported to exhibit 
reduced pathogenicity [11]. Distribution of T4SS was sporadic among Pectobacterium genomes and the T4SS-deficient strains were present among all five Pectobacterium species ( $P$. carotovorum, $P$. polaris, $P$. atrosepticum, $P$. parmentieri, Candidatus $P$. maceratum) as well as D. paradisiaca Ech703 (Fig. 5b). The copy number also varied among the strains that carried it. For example, two copies of T4SS (BASYS00894-BASYS00885 and BASYS00696-BASYS00686), were identified in three $P c b$ strains (BC1, BZA12, and BD255), three Pco strains (Q115, Q142, and Q166), three Pca strains (ICMP 19972, ICMP 19971, and KKH3), as well as D. zeae Ech586, while the other strains only possessed a single copy.

\section{A novel T6SS type (T6SSc) present in Pco strains}

Our analysis of 84 Pectobacterium genomes identified three types of T6SSs (T6SSa-c), of which T6SSc is a novel type that identified and termed in this study (Fig. 5a and Additional file 1: Table S7). T6SSa was absent in P. polaris NCPPB 3395, but present in the rest of the $P$. polaris strains as well as the other four species (P. atrosepticum, P. parmentieri, P. polaris, Candidatus P. maceratum, and $P$. carotovorum). Similar to T1SS, T3SS, and T4SS, we found that the T6SS gene cluster was not present in the D. paradisiaca Ech703 genome, a finding consistent with results from a study by Zhou et al. [45]. T6SSb was a specific cluster that was only occurred in (all of the) P. parmentieri strains. Interestingly, we also found a novel T6SS that we termed T6SSc (BCS714475-BCS714610) which was only present in the Pco strains; the distribution of each of the genes of the T6SSc cluster varied among the Pco strains (Fig. 6 and Additional file 1: Table S8): nine genes were present in all 15 Pco strains, while the remaining 19 individual genes were variously present among the strains we surveyed. Similarity analysis based on DNA sequences revealed high genetic variation in pairwise identity comparisons of T6SSc between strains, ranging from $40.1 \%$ for the most divergent T6SSc of strains to $100 \%$ similarity for the most conserved.

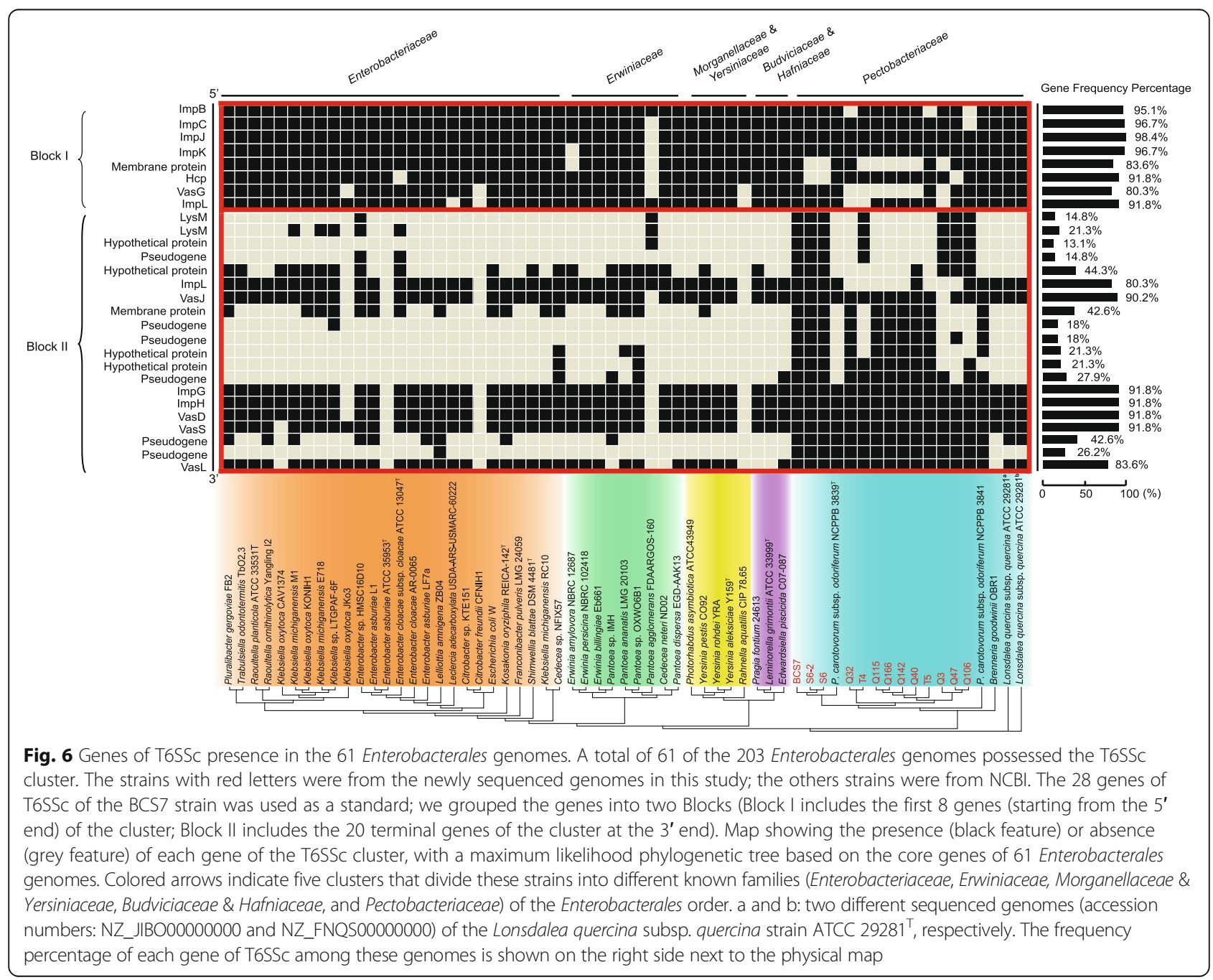




\section{An expanded examination of the novel T6SSc and the $s r l$ operon}

Based on the results of our comparative genomics study of $P$. carotovorum subspecies and the other four closely related species of Pectobacterium, we found that the novel cluster T6SSc and the $s r l$ operon were specific to Pco subspecies. To evaluate the distribution of this operon and T6SSc in other closely related strains, we conducted an expanded genome (203 genomes, Additional file 1: Table S8-S9) analysis with all available genomes data of Pectobacterium downloaded from GenBank in July 2018 as well as other sequences retrieved from GenBank for representative strains from each branch of the phylogenetic trees of the Enterobacterales order from a study by Adeolu et al. [46].

\section{The highly conserved srl operon in Pco subspecies}

A total of 203 genomes of Enterobacterales strains were analyzed: only 66 of them possessed this $\mathrm{srl}$ operon (Additional file 1: Table S9). In addition to all of the sequenced Pco strains (including 13 from this study and the 2 from NCBI), we did not find any other Pectobacterium spp. or Dickeya spp. which cause similar soft rot diseases that harbored this operon. Besides the Pco strains, the srl operon was also present in two sequenced Brenneria strains and in one of the three sequenced Sodalis strains of the Pectobacteriaceae family. The $s r l$ operon occurred among most genomes of the Enterobacteriaceae (81.1\%), Yersiniaceae (80.0\%), and Erwiniaceae (62.5\%) families, but is not present in the Morganellaceae, Budviciaceae, or Hafniaceae families. We found some plant pathogens with a broad host range (e.g., Pantoea ananatis) [47], some that infect hosts containing high levels of sorbitol (e.g., Erwinia spp.) [48-50], and some growth-promoting rhizobacteria (e.g., nitrogen-fixing strain) [51] also possess this operon. In addition, we aligned the $\mathrm{srl}$ operon sequences (protein and DNA) from these 66 strains and conducted phylogenetic and similarity (MUSCLE) analyses. The similarity analysis (protein and DNA) revealed few genetic differences (pairwise similarity $>99.4 \%$ in protein sequences; pairwise similarity $>99.5 \%$ in DNA sequences) in the $s r l$ operon between different Pco strains. Phylogenetic analysis based on DNA and protein sequences of the srl operon (Additional file 3: Figure S2) both showed that Pco strains were clustered into the clade with Erwinia spp., but separated from the clades containing Enterobacteriaceae spp. and Yersiniaceae spp. This result was consistent with the $s r l$ operon of Pco strains sharing highest similarity with those of Erwinia spp. (69.1-71.3\% in DNA sequence and $78.6-80.0 \%$ in protein sequence).

\section{Genetic variation of the novel secretion system T6SSc} Sixty-one Of the 203 Enterobacterales genomes we examined possessed the T6SSc cluster. In contrast to the high conservation of the srl operon among Pco strains, relatively high genetic variation was observed among the Pco T6SSc cluster sequences. Despite of this, the phylogenetic trees (not shown) built based on their T6SSc cluster sequences (protein and DNA) clustered all of the 15 Pco strains sequenced to date into a clade which was clearly separated from the other Enterobacterales strains. The T6SSc cluster was also present in a limited number of Brenneria goodwinii and Lonsdalea quercina strains in the Pectobacteriaceae, as well as being present sporadically among the other 6 families of the Enterobacterales order (Additional file 1: Table S8).

An analysis that screened for the presence/absence of individual genes of the T6SSc cluster in 61 Enterobacterales genomes showed two clear distribution patterns (Fig. 6). It suggested that the cluster consisted of two blocks of genes: the first, Block I, includes the first 8 genes (starting from the $5^{\prime}$ end) of the cluster, while Block II includes the 20 terminal genes of the cluster at the 3' end. We found that genes of Block I were more highly conserved than those of Block II, a finding that merits further study that may help to clarify the evolutionary history of Pco species within the Pectobacterium genus.

\section{Discussion}

Historical assessments which assert that Pcc has the widest host range among the Pectobacterium taxa are due for revision, considering that multiple studies which examined the pathogenicity and host ranges of $P c b$ and Pco have now established a broad spectrum of hosts for these subspecies as well $[5,52,53]$. Many strains previously known as Pcc have also been re-classified into other species in recent years $[8,9]$. Our finding that the $P$. peruviense, $P$. polaris, and Candidatus P. maceratum strains did not possess either the T6SSc cluster or the $s r l$ operon, viewed alongside our finding that no Pcc strains harbored either cluster, are consistent with the recently proposed adjustments to the classification of Pectobacterium species, which have posited that the newly proposed species are derived from Pcc. We also noticed that the genomic traits of $P c a$ for some pathogenicity-related genes as well as the CRISPR-Cas systems, were not consistent with any of the three-known Pcc, Pcb and Pco subspecies, which is further support to the new subspecies proposal.

Interestingly, an $\mathrm{srl}$ operon related to sorbitol metabolism was specific to Pco genomes among the Pectobacterium genus based on the BLAST analysis using both DNA and amino acid sequences. Other studies have reported that mutants with loss-of-function for this operon in another phytopathogen (Erwinia amylovora) with a sorbitol deficiency were unable to grow on minimal medium with sorbitol [26]. Our biochemical test results also confirmed that these Pco strains with the $s r l$ 
operon could produce acid from sorbitol or could utilize this carbon source, while other strains lacking this operon like $P c c$ and $P c b$ could not. This is further supported by some previous studies which showed that Pectobacterium strains and the type strains of other species, such as $P c c$, $P$. wasabiae, $P$. betavasculorum, and P. atrosepticum could not utilize/produce acid from sorbitol but that the type Pco strain as well as other Pco strains could [54-57]. However, the ability of these Pectobacterium type strains to use sorbitol is still obscure; there are conflicting reports about the biochemical phenotypes for these strains reported in various studies $[8,52,58]$.

Moreover, although the sequences of the $s r l$ operon from Pco strains are conserved, and are closely related to those present in Erwinia spp., whether the Pco strains acquired this operon from Erwinia spp., or if they acquired it from a common ancestor (and may have subsequently lost it in certain strains) still awaits clarification. Further, although this operon is strictly required for the pathogenicity of E. amylovora [26], its function in Pco is still unknown; it is clearly important considering its high level of conservation in Pco strains.

The other specific cluster to Pco subspecies among Pectobacterium is T6SSc; while the T6SSa and T6SSb type clusters have been reported extensively, we are unaware of other studies reporting a cluster with the gene order we detected in this T6SSc type cluster. T6SS have been shown to function in host cell adherence and in competition with other microbes, but the role of this class of gene cluster in Pectobacterium during infection remains unclear [2]. Our results confirmed the previously reported finding that $P$. atrosepticum contains only one T6SS (T6SSa), whereas $P$. parmentieri harbors two such machineries (T6SSa and T6SSb) [22]. However, in contrast to the previous description that $P$. carotovorum stains only have one T6SS (T6SSa), we detected T6SSc in these species. Although the components of this system were not strictly consistent among the Pco strains, and its function and potential targets must of course be further determined, it will be interesting to explore the possible functional contribution of this novel T6SSc to the pathophysiology of soft rot diseases. Many other genetic variations including pathogenicity-related genes as well as CRISPR-Cas systems that were revealed among the subspecies and species by this study lay a foundation for future work to elucidate the genetic basis of pathogenicity in Pectobacterium. It will also be interesting to determine which among these genomic variations in the different species/subspecies contribute to host ranges and geographic distributions.

\section{Conclusion}

Our findings significantly expand the body of knowledge surrounding the genetic basis for pathogenicity of soft rot bacteria in general. The genetic variations catalogued in this study also provide new insights into the evolutionary history of Pectobacterium and give extensive definitive evidence for accurately classifying members of the Pectobacterium species and subspecies. These analyses will provide a foundation for generating hypotheses about pathogenicity, host-bacteria interactions, taxonomy, and phylogenetic relationships among soft rot bacteria and other phytopathogens.

\section{Methods}

Collection and phenotyping of 32 Chinese Pectobacterium carotovorum isolates

Thirty-two strains from 3 economically important Chinese vegetables, Chinese cabbage (Brassica rapa subsp. pekinensis), bok choy (Brassica rapa subsp. chinensis), and celery (Apium graveolens) were collected from North China (Beijing area). Typical symptoms of soft rot were observed on the petioles and stems of these plants, which were grown in cultivated field in different districts of Beijing (Additional file 1: Table S3). Isolation of bacteria from diseased samples was carried out according to previous studies [54], were identified by $\mathrm{Yu}$ Tian and Xiaoying Li, previously, and were preserved in Beijing Key Laboratory of Agricultural Genetic Resources and Biotechnology, Beijing Academy of Agriculture and Forestry Science. These strains were tested for pathogenicity by inserting one pipette tip containing $10 \mu \mathrm{L}$ of bacterial suspension $\left(2 \times 10^{8} \mathrm{CFU} / \mathrm{mL}\right) 2 \mathrm{~mm}$ into each sterilized Chinese cabbage petioles surface with sterile distilled water as control. The petioles were then incubated at $28{ }^{\circ} \mathrm{C}$ and $95 \%$ relative humidity for $24 \mathrm{~h}$ before the diameter of the rotting tissue in each petiole was measured. Each strain was represented by three replicate petioles, and the experiment was performed three times. The data were subjected to one-way ANOVAs using SPSS 10.0. Means were compared by the Duncan's tests, and statistical significance was determined at 5\% levels. Strains were re-isolated successfully from symptomatic Chinese cabbages to complete Koch's postulates. All strains were assayed with several biochemical tests: reducing substances from sucrose; acid production from D-sorbitol, D-arabitol, palatinose, and $\alpha$-methylglucoside; utilization of citrate, and growth in $5 \% \mathrm{NaCl}$ and at $37^{\circ} \mathrm{C}$ [59]. Moreover, the 94 phenotypes (the oxidation of the 71 carbon sources and chemical susceptibility to 23 compounds) were analyzed using GEN III OmniLog system by the BIOLOG ${ }^{\mathrm{mm}}$ GEN III microplates [60].

Genome sequencing, assembly, and annotation of the $\mathbf{3 2}$ newly isolated Pectobacterium carotovorum strains

Genomic DNA was extracted and purified using an EasyPure Genomic DNA Kit (Transgen, China) according to the product instructions. Sequencing of the $P c b \mathrm{BC} 1$ 
genome (accession: CP009769) was performed using the Illumina Hiseq 2000 platform with $2 \mathrm{~Kb}$ mate pairs and 200 to $500 \mathrm{bp}$ unique paired-ends insert size libraries. The $\mathrm{BC} 1$ sequence was assembled by SOAPdenovo 2.0 software representing a 335-fold average genomic coverage. The scaffold order was based on the PCC21 reference genome, and a PCR-based genomic walking method was used to fill gaps between scaffolds. Sequencing of the Pco BCS7 genome (accession: CP009678) was performed with Pacific Biosciences RS II sequencing technology. A $10 \mathrm{~Kb}$ Single-Molecule Real-Time (SMRT) library was prepared from sheared genomic DNA using a 10-kb template library preparation workflow. The library was sequenced on 2 SMRT cells, providing about 137 -fold genome coverage. The denovo assembly of the BCS7 reads was performed with the Hierarchical Genome Assembly Process (HGAP) algorithm in SMRT Portal (version 2.3.0). The genomes of the additional $30 \mathrm{P}$. carotovorum strains were sequenced with MPS (massively parallel sequencing) Illumina technology. A paired-end DNA library with an insert size of $500 \mathrm{bp}$ was constructed and was sequenced using an Illumina HiSeq. Quality control of paired-end reads was performed using an in-house program to filter the low-quality reads, Illumina PCR adapter reads, and duplication reads. After filtering, all 30 of the strains had the sequencing coverage between 65 -fold and 200-fold. Denovo assembly of the reads was performed with the SOAPdenovo algorithm (http://soap.genomics.org.cn/soapdenovo.html). All reads were used for further gap closure.

Coding genes of these 32 newly sequenced strains were predicted with GeneMark software by the self-training program GeneMarkS (version: 4.6b, http://topaz.gatech. edu/GeneMark/). Transfer RNA (tRNA) genes were predicted with tRNAscan-SE (version: 1.3.1, http:/gtrnadb. ucsc.edu/) [61], Ribosome RNA (rRNA) genes were predicted with rRNAmmer [62], and sRNAs were predicted by BLAST against the Rfam database. Long terminal repeats sequences of all the tested genomes in this study were predicted using Repeat Masker (http://www. repeatmasker.org/) and tandem repeats were analyzed using Tandem Repeat Finder (http://tandem.bu.edu/trf/ trf.html). Among these tandem repeats, arrays with repeat units $15 \mathrm{bp}$ to $65 \mathrm{bp}$ will be considered as minisatellites DNA. Gene functional annotations of each of the 32 genomes was performed by BLAST analysis against multiple databases, including NCBI NR (Version: 20150405, http://www.ncbi.nlm.nih.gov/), KEGG (Version: 59, http://www.genome.jp/kegg/), COG (Version: 20090331, http://www.ncbi.nlm.nih.gov/COG/), GO (Version: 20150405, http://geneontology.org/), SwissProt (Version: 20150414, http://www.ebi.ac.uk/uniprot/), PHI (Version: 3.6, http://www.phi-base.org/), VFDB (Version: Tue May 5 10:06:01 2015, http://www.mgc.ac.cn/ VFs/main.htm), MvirDB database [63], ARDB (Version:
1.1, http://ardb.cbcb.umd.edu/), CARD (Version: 20140415, http://arpcard.mcmaster.ca/), CAZy (Version: 20141020, http://www.cazy.org/), TCDB (Version: 20110715, http://www.tcdb.org/). For each BLAST hit (a given ORF), the best alignment with the lowest e-value and highest score was retained; to be retained, a hit required at least 0.4 identity for a sequence at $40 \%$ of a given alignment's length. All 32 genomes newly sequenced in this study have been deposited in the NCBI genome database (Accession numbers were shown in Additional file 1: Table S3).

\section{Comparative genomic analysis}

A comprehensive comparative genomics study of $84 \mathrm{Pec}$ tobacterium genomes (including the 32 newly sequenced genomes in this study and 52 other Pectobacterium genomes published in GenBank by July 2018, for strains including $P$. carotovorum, $P$. polaris, Candidatus $P$. maceratum, $P$. atrosepticum, and $P$. parmentieri) was conducted using three complete Dickeya genomes (D. zeae Ech586, D. paradisiaca Ech703, and D. dadantii 3937) as the reference outgroup. Note that the previously reported P. carotovorum strain 3-2, as well as the Pcc strains ATCC 39048 and Ecc71, were reclassified in this study (Fig. 2a); therefore, strains Ecc71 and 3-2 were part of the Candidatus P. maceratum group and strain ATCC 39048 was part of the $P c b$ group in each of the bioinformatics analyses of this in this comparative genomics study. The verified classification and detailed information for each of the strains are shown in Additional file 1: Table S3. All of these 84 Pectobacterium genomes were used for the following bioinformatics analyses.

\section{Whole-genome SNP detection and identity score analyses}

Genome-Wide SNP detection was performed using MUMmer comparison software: the whole-genome sequence of $\mathrm{BC} 1$ (the reference) was compared with genome sequence of the other strains to identify differences. BLAT (BLAST-Like Alignment Tool) software was then used to compare the sequences adjacent to the SNP loci to verify a given SNP's validity [64]. Repeat regions were detected by BLAST, Repeat Masker, and Tandem Repeats Finder software. If the length of the SNP adjacent sequence alignment in the BLAT results was less than $101 \mathrm{bp}$, or if a SNP was found in a repeat region, the data was considered as suspect and was removed. We performed a phylogenetic analysis based on SNP data: for all species, all SNPs were joined in the same order. PhyML software (Version: 3.0, http://www.atgc-montpellier.fr/ phyml/) was then used to construct the phylogenetic tree with a maximum likelihood model. The number of bootstrap replications was set to 1000 .

To identify genetic relatedness in any two strains, pairwise Identity Scores (IS) were calculated [65]. The IS is 
the ratio of unique SNPs between two accessions; this was determined using an in-house Python script and visualized with a heat map which produced with the ggplot2R package.

\section{Pan-genome analyses}

Eighty-four Pectobacterium genomes, including $56 P$. carotovorum, 6 P. parmentieri, 10 P. atrosepticum, 8 Candidatus P. maceratum, and $4 \mathrm{P}$. polaris genomes were used in the pan-genome analysis of four closely related species of the Pectobacterium genus (Additional file 1: Table S3). This included the 9 Pcc, 29 Pcb, 15 Pco, and $3 P c a$ genomes that were used in the aforementioned pan-genome analysis of the $P$. carotovorum species. Subspecies core orthologous genes and strain-specific unique genes were also examined in the $9 P c c, 15 P c o, 29 P c b$, and $3 P c a$ genomes sequences, respectively. Specifically, it should be noted that the five pan-genomes of each species (that is, the pan-genome for $56 P$. carotovorum genomes, that of the 6 P. parmentieri, $10 P$. atrosepticum, 8 Candidatus $\mathrm{P}$. maceratum, and 4 P. polaris genomes, respectively) were used as the petals for generating the Venn diagram for the Pectobacterium genus.

Likewise, the pan-genomes for each of the four subspecies (that is $29 P c b, 3 P c c, 15 P c o$, and $3 P c a$ genomes, respectively) were used as the petals to generate the Venn diagram for the $P$. carotovorum species. The core and pan genes identified and clustered using CD-HIT rapid clustering of similar proteins software $[66,67]$, with a threshold of $70 \%$ pairwise identity and a 0.6 length difference cut-off in amino acid sequences. Genes that did not reach the threshold were used to initiate a new cluster. Genes from multiple species in each core gene cluster were aligned to each other to make a multi-sequence alignment using MUSCLE (Version: 3.8.31, http://www.drive5.com/muscle). The CD-HIT analysis also identified the genes that were unique to each species/subspecies of Pectobacterium, and their functions differences based on COG annotations were performed. Finally, a core-gene phylogenetic tree of these Pectobacterium strains was constructed with three Dickeya strains as the outgroup. The PhyML (Version: 3.0, http://www. atgc-montpellier.fr/phyml/) software was used with a maximum likelihood model. The number of bootstrap replications was set to 1000 .

\section{Gene cluster analysis of orthologs}

BLAST analysis using both DNA and amino acid sequences was used to identify the presence of homologs of previously known genes (e.g., pathogenicity-related genes) in the genomes in this study. For each BLAST hit (a given ORF), the best alignment with the lowest e-value and highest score was retained; to be retained, a hit required at least a 0.4 identity for a sequence at $40 \%$ of a given alignment's length. Detection of CRISPR-Cas system components. The CRISPR Finder online tool (http://crispr.i2bc.paris-saclay.fr/Server/) was used to identify all of the predicted CRISPR components for each strain. Predicted cas genes were also identified with BLAST analysis according to Makarova (2011) [68]. The CRISPR and cas results were combined to determine the predicted locations of the CRISPR-Cas system(s) in each strain.

\section{An expanded examination of the novel T6SSc and the srl operon}

After confirming the $s r l$ operon and T6SSc were two Pco-specific clusters within 84 Pectobacterium and 3 reference Dickeya genome sequences tested above, we examined its distribution in the Pectobacterium genus and even in the order Enterobacterales. This analysis included the 203 genomes, including all of the available Pectobacterium downloaded from GenBank in July 2018, as well as other sequences from GenBank for representative strains from each branch of the phylogenetic trees of Enterobacterales as reported by Adeolu et al. [46]. These 203 Enterobacterales genomes covered 62 species of 31 genera across 7 families (Enterobacteriaceae, Erwiniaceae, Morganellaceae, Yersiniaceae, Budviciaceae, Hafniaceae, and Pectobacteriaceae) of the Enterobacterales order. BLAST analysis using both DNA and amino acid sequences was used to identify the presence of homologs of the genes of the $\mathrm{srl}$ operon and T6SSc cluster in these 203 Enterobacterales genomes with the sequences of the BCS7 strain (the only Pco strain with a complete genome) used as a standard. After identifying homologs in these strains, we examined the similarity of the sequences for these two clusters using a maximum likelihood phylogenetic tree based on both the DNA and amino acid sequences. We also calculated the ratio of the similarity based on the total length of alignment of each couple strains in the matrix based on the results of multi-sequence alignment (both the DNA and amino acid sequences) using MUSCLE software.

\section{Additional files}

\footnotetext{
Additional file 1: Table S1. The biochemical characters and pathogenicity of 32 Chinese Pectobacterium carotovorum strains. Table S2. Selected phenotypic characteristics of Pectobacterium carotovorum subsp. brasiliense, $P$. carotovorum subsp. carotovorum and $P$. carotovorum subsp. odoriferum. Table S3. Eighty-four Pectobacterium genomes used in this study, with accession number, host origin and location information. Table S4. The general genomic features of the 84 Pectobacterium genomes. Table S5. The CRISPR-Cas systems identified in the 84 Pectobacterium genomes. Table S6. Presence of genes encoding general virulence factors in the 84 Pectobacterium genomes. Table S6-1. The position of $r m s B$, the RNA fragment related to virulence in the 84 Pectobacterium genomes. Table S7. Gene clusters for TNSSs identified in the 84 Pectobacterium genomes. Table S8. Presence of the T6SSc gene cluster in the 203 Enterobacterales genomes. Table S9. Presence of the $\mathrm{srl}$ operon in the 203 Enterobacterales genomes. (XLSX $751 \mathrm{~kb})$
} 
Additional file 2: Figure S1. Soft rot symptoms in Brassica rapa subsp. pekinensis (a), Brassica rapa subsp. chinensis (b), and Apium graveolens (c) (PDF $112808 \mathrm{~kb}$ )

Additional file 3: Figure S2. Phylogenetic tree based on the amino acid sequences of the $\mathrm{srl}$ operon in the 66 Enterobacterales strains. A total of 66 of the 203 Enterobacterales genomes were found to possess the srl operon in this study. The phylogenetic trees based on DNA and amino acid sequences were almost identical. The strains with red letters were the newly sequenced in this study. They were clustered into the clade containing the Ewinia strains. The tree was constructed using a maximum likelihood method and was generated with 1000 bootstrap replicates. (PDF $763 \mathrm{~kb}$ )

\section{Abbreviations}

BLAT: BLAST-like alignment tool; COG: Clustering of orthologous groups; CRISPR-Cas: Clustered regularly interspaced palindromic repeats/CRISPRassociated proteins; ECA: Enterobacterial common antigen; IS: Identity scores; LPS: Lipopolysaccharide; Nip: Necrosis-inducing protein; Pap: Pyelonephritisassociated pili; Pca: $P$. carotovorum subsp. actinidiae; $P c b$ : P. carotovorum subsp. brasiliense; Pcc: P. carotovorum subsp. carotovorum; Pco: $P$. carotovorum subsp. odoriferum; PCWDEs: Plant cell wall-degrading enzymes; PGAAP: Prokaryotic Genome Annotation Pipeline; SNPs: Single nucleotide polymorphisms; Svx: A homologue of an avirulence protein of Xanthomonas; TNSS: Protein secretion system

\section{Acknowledgements}

Not applicable.

\section{Funding}

This work was supported by grants from the Earmarked Fund for Beijing Innovation Consortium of Agriculture Research System (BAIC07). The funding source supported the publication of this work and data analysis while had no role in study design and data collection, interpretation of data and in writing the manuscript.

\section{Availability of data and materials}

All data generated or analyzed during this study are included in this published article [and its additional information files]. All genome sequences used in the course of this study are available from the National Centre for Biotechnology Information, (NCBI) genome database (https://www.ncbi.nlm.nih.gov). Additionally, all genomes sequenced during the course of this study have been deposited in the NCBI genome database under the following accession numbers: CP009769, CP009678, JUJM00000000, JUJJ00000000, JUJK00000000, JUJL00000000, JUJH00000000, JUJB00000000, JUJC00000000, JUJP00000000, JUJD00000000 JUJE00000000, JUJN00000000, JUJF00000000, JUJT00000000, JUJO00000000, JUJQ00000000, JUJG00000000, JUJR00000000, JUJS00000000, MTAG00000000, MTAI00000000, MTAH00000000, MTAJ00000000, MTAK00000000, MTAL00000000, MTAO00000000, MTAP00000000, MTAN00000000, MTAR00000000, MTAQ000 00000, MTAM00000000. Biological materials from the current study are available from the corresponding author on reasonable request.

\section{Authors' contributions}

$\mathrm{HX}$ designed the experiment and revised the manuscript; $X Y L, Y L M$, and $S Q L$, performed the molecular, biochemical data collection and bioinformatics analysis; XYL, YLM, and SQL wrote the manuscript. SJY, SSX, and YT revised the manuscript. All authors read and approved the final manuscript.

\section{Ethics approval and consent to participate}

This study did not involve humans, human data or animals; no ethics approval or consent is required to publish the results.

\section{Consent for publication}

Not applicable.

\section{Competing interests}

The authors declare that the research was conducted in the absence of any commercial or financial relationships that could be construed as a potential conflict of interest.

\section{Publisher's Note}

Springer Nature remains neutral with regard to jurisdictional claims in published maps and institutional affiliations.

\section{Author details}

${ }^{1}$ Beijing Agro-Biotechnology Research Center, Beijing Academy of Agriculture and Forestry Sciences, Beijing 100097, People's Republic of China. ${ }^{2}$ Beijing Key Laboratory of Agricultural Genetic Resources and Biotechnology, Beijing 100097, People's Republic of China. ${ }^{3}$ Health Time Gene Institute, Shenzhen, Guangdong 518000, People's Republic of China.

Received: 8 June 2018 Accepted: 19 November 2018 Published online: 07 December 2018

\section{References}

1. Onkendi EM, Moleleki LN. Characterization of Pectobacterium carotovorum subsp. carotovorum and brasiliense from diseased potatoes in Kenya. Eur J Plant Pathol. 2014:139:557-66.

2. Davidsson PR, Kariola T, Niemi O, Palva ET. Pathogenicity of and plant immunity to soft rot pectobacteria. Front Plant Sci. 2013:4:191.

3. Khayi S, Cigna J, Chong TM, Quêtu-Laurent A, Chan KG, Hélias V, et al Transfer of the potato plant isolates of Pectobacterium wasabiae to Pectobacterium parmentieri sp. nov. Int J Syst Evol Microbiol. 2016;66:5379-83.

4. Czajkowski $R$, Pérombelon $M$, Jafra $S$, Lojkowska $E$, Potrykus $M$, van der Wolf $\mathrm{JM}$, et al. Detection, identification and differentiation of Pectobacterium and Dickeya species causing potato blackleg and tuber soft rot: a review. Ann Appl Biol. 2015;166:18-38.

5. Nabhan S, de Boer SH, Maiss E, Wydra K. Taxonomic relatedness between Pectobacterium carotovorum subsp. carotovorum, Pectobacterium carotovorum subsp. odoriferum and Pectobacterium carotovorum subsp. brasiliense subsp. nov. J Appl Microbiol. 2012:113:904-13.

6. Koh YJ, Kim GH, Lee YS, Sohn SH, Koh HS, Kwon S, et al. Pectobacterium carotovorum subsp. actinidiae subsp. nov., a new bacterial pathogen causing canker-like symptoms in yellow kiwifruit, Actinidia chinensis. New Zeal J Crop Hort. 2012:40:269-79.

7. Nabhan S, Boer SHD, Maiss E, Wydra K. Pectobacterium aroidearum sp. nov., a soft rot pathogen with preference for monocotyledonous plants. Int J Syst Evol Micr. 2013;63:2520-5.

8. Waleron M, Misztak A, Waleron M, Franczuk M, Wielgomas B, Waleron K Transfer of Pectobacterium carotovorum subsp. carotovorum strains isolated from potatoes grown at high altitudes to Pectobacterium peruviense sp. nov. Syst Appl Microbiol. 2017;41:85-93.

9. Dees MW, Lysøe E, Rossmann S, Perminow J, Brurberg MB. Pectobacterium polaris sp. nov. isolated from potato (Solanum tuberosum). Int J Syst Evol Microbiol. 2017;67:5222-9.

10. Shirshikov FV, Korzhenkov AA, Miroshnikov KK, Kabanova AP, Barannik AP, Ignatov AN, et al. Draft genome sequences of new genomospecies "Candidatus Pectobacterium maceratum" strains, which cause soft rot in plants. Genome Announc. 2018;6:e00260-18.

11. Bell KS, Sebaihia M, Pritchard L, Holden MTG, Hyman LJ, Holeva MC, et al. Genome sequence of the enterobacterial phytopathogen Erwinia carotovora subsp. atroseptica and characterization of virulence factors. Proc Natl Acad Sci U S A. 2004;101:11105-10.

12. Richter C, Fineran PC. The subtype I-F CRISPR-Cas system influences pathogenicity island retention in Pectobacterium atrosepticum via crRNA generation and Csy complex formation. Biochem Soc T. 2013;41:1468-74.

13. Nikolaichik Y, Gorshkov V, Gogolev Y, Valentovich L, Evtushenkov A. Genome sequence of Pectobacterium atrosepticum strain 21A. Genome Announc. 2014:2:e00935-14.

14. Koskinen JP, Laine $P$, Niemi $O$, Nykyri J, Harjunpää H, Auvinen $P$, et al. Genome sequence of Pectobacterium sp. strain SCC3193. J Bacteriol. 2012; 194:6004

15. Park TH, Choi BS, Choi AY, Choi IY, Heu S, Park BS. Genome sequence of Pectobacterium carotovorum subsp. carotovorum strain PCC21, a pathogen causing soft rot in Chinese cabbage. J Bacteriol. 2012;194:6345-6.

16. Niemi $\mathrm{O}$, Laine $\mathrm{P}$, Koskinen $\mathrm{P}$, Pasanen $\mathrm{M}$, Pennanen $V$, Harjunpää $\mathrm{H}$, et al. Genome sequence of the model plant pathogen Pectobacterium carotovorum SCC1. Stand Genomic Sci. 2017;12:87

17. Huang YF, Liu CY, Wang H, Guan TS, Liu L, Yu SY. Bioinformatic analysis of the complete genome sequence of Pectobacterium carotovorum subsp. 
brasiliense BZA12 and candidate effector screening. J Plant Pathol. 2018. https://doi.org/10.1007/s42161-018-0126-7.

18. Toth IK, Pritchard L, Birch PR. Comparative genomics reveals what makes an enterobacterial plant pathogen. Annu Rev Phytopathol. 2006:44:305-36.

19. Ma B, Charkowski AO, Glasner JD, Perna NT. Identification of host-microbe interaction factors in the genomes of soft rot-associated pathogens Dickeya dadantii 3937 and Pectobacterium carotovorum WPP14 with supervised machine learning. BMC Genomics. 2014;15:508.

20. Toth I, Humphris S, Campbell E, Pritchard L. Why genomics research on Pectobacterium and Dickeya makes a difference. Am J Potato Res. 2015;92:218-22.

21. Glasner JD, Marquez-Villavicencio M, Kim HS, Jahn CE, Ma B, Biehl BS, et al. Niche-specificity and the variable fraction of the Pectobacterium pangenome. Mol Plant-Microbe Interact. 2008;21:1549-60.

22. Nykyri J, Niemi O, Koskinen P, Noksokoivisto J, Pasanen M, Broberg M, et al. Revised phylogeny and novel horizontally acquired virulence determinants of the model soft rot phytopathogen Pectobacterium wasabiae SCC3193. PLoS Pathog. 2012;8:e1003013.

23. Mcewan CEA, Gatherer D, Mcewan NR. Nitrogen-fixing aerobic bacteria have higher genomic GC content than non-fixing species within the same genus. Hereditas. 1998;128:173-8.

24. Visnovsky SB, Panda P, Taylor R, Pitman AR. Draft genome sequences of Pectobacterium carotovorum subsp. actinidiae ICMP 19971 and ICMP 19972, two strains isolated from Actinidia chinensis with symptoms of summer canker in South Korea. Genome Announc. 2017;5:e00104-17.

25. Xu Y, Zhu Y, Wang Y, Chang YF, Zhang Y, Jiang X, et al. Whole genome sequencing revealed host adaptation-focused genomic plasticity of pathogenic Leptospira. Sci Rep. 2015;6:20020.

26. Aldridge P, Metzger M, Geider K. Genetics of sorbitol metabolism in Erwinia amylovora and its influence on bacterial virulence. Mol Gen Genomics. 1997; 256:611-9.

27. Przybilski R, Richter C, Gristwood T, Clulow JS, Vercoe RB, Fineran PC. Csy4 is responsible for CRISPR RNA processing in Pectobacterium atrosepticum. RNA Biol. 2011;:8:517-28.

28. Melvin JA, Scheller EV, Noël CR, Cotter PA. New insight into filamentous hemagglutinin secretion reveals a role for full-length FhaB in Bordetella virulence. MBio. 2015;6:e01189-15.

29. Ling J, Pan H, Gao Q, Xiong L, Zhou Y, Zhang D, et al. Aerobactin synthesis genes iucA and iucC contribute to the pathogenicity of avian pathogenic Escherichia coli O2 strain E058. PLoS One. 2013;8:e57794.

30. Lee HS, Abdelal AH, Clark MA, Ingraham JL. Molecular characterization of nosA, a Pseudomonas stutzeri gene encoding an outer membrane protein required to make copper-containing $\mathrm{N}_{2} \mathrm{O}$ reductase. J Bacteriol. 1991;173: 5406-13.

31. Båga M, Normark S, Hardy J, O'Hanley P, Lark D, Olsson O, et al. Nucleotide sequence of the papA gene encoding the pap pilus subunit of human uropathogenic Escherichia coli. J Bacteriol. 1984;157:330-3.

32. Kariyawasam S, Johnson TJ, Nolan LK. The pap operon of avian pathogenic Escherichia coli strain 01:K1 is located on a novel pathogenicity island. Infect Immun. 2006;74:744-9.

33. Köiv V, Andresen L, Mäe A. AepA of Pectobacterium is not involved in the regulation of extracellular plant cell wall degrading enzymes production. Mol Gen Genomics. 2010;283:541-9.

34. Chang JH, Desveaux D, Creason AL. The ABCs and 123s of bacterial secretion systems in plant pathogenesis. Phytopathology. 2014;52:317-45.

35. Izadi N, Henry Y, Haladjian J, Goldberg ME, Wandersman C, Delepierre M, et al. Purification and characterization of an extracellular heme-binding protein, HasA, involved in heme iron acquisition. Biochemistry. 1997;36: 7050-7.

36. Delepelaire P. Type I secretion in gram-negative bacteria. Biochim Biophys Acta. 2004;1694:149-61.

37. Marits R, Kõiv V, Laasik E, Mäe A. Isolation of an extracellular protease gene of Erwinia carotovora subsp. carotovora strain SCC3193 by transposon mutagenesis and the role of protease in phytopathogenicity. Microbiology. 1999;145:1959-66.

38. Korotkov KV, Sandkvist M, Hol WG. The type II secretion system: biogenesis, molecular architecture and mechanism. Nat Rev Microbiol. 2012;10:336-51.

39. Ferrandez $Y$, Condemine $G$. Novel mechanism of outer membrane targeting of proteins in gram-negative bacteria. Mol Microbiol. 2008;69:1349-57.

40. Charkowski A, Blanco C, Condemine G, Expert D, Franza T, Hayes C, et al. The role of secretion systems and small molecules in soft-rot Enterobacteriaceae pathogenicity. Phytopathology. 2012;50:425-49.
41. Pirhonen M, Saarilahti H, Karlsson MB, Palva ET. Identification of pathogenicity determinants of Erwinia carotovora subsp. carotovora by transposon mutagenesis. Mol Plant Microbe In. 1991;4:276-83.

42. Holeva MC, Bell KS, Hyman LJ, Avrova AO, Whisson SC, Birch PR, et al. Use of a pooled transposon mutation grid to demonstrate roles in disease development for Erwinia carotovora subsp. atroseptica putative type III secreted effector (DspE/a) and helper (HrpN) proteins. Mol Plant Microbe In. 2004;17:943-50.

43. Kim HS, Ma B, Perna NT, Charkowski AO. Phylogeny and virulence of naturally occurring type III secretion system-deficient Pectobacterium strains. Appl Environ Microb. 2009;75:4539-49.

44. Josenhans C, Suerbaum $S$. The role of motility as a virulence factor in bacteria. Int J Med Microbiol. 2002;291:605-14.

45. Zhou J, Cheng Y, Lv M, Liao L, Chen Y, Gu Y, et al. The complete genome sequence of Dickeya zeae EC1 reveals substantial divergence from other Dickeya strains and species. BMC Genomics. 2015;16:571.

46. Adeolu M, Alnajar S, Nausha S, Gupta RS. Genome based phylogeny and taxonomy of the "Enterobacteriales": proposal for Enterobacterales ord. nov. divided into the families Enterobacteriaceae, Erwiniaceae fam. nov. Pectobacteriaceae fam. nov., Yersiniaceae fam. nov., Hafniaceae fam. nov., Morganellaceae fam. nov., and Budviciaceae fam. nov. Int J Syst Evol Microbiol. 2016;66:5575-99.

47. Weller-Stuart T, Toth I, de Maayer P, Coutinho T. Swimming and twitching motility are essential for attachment and virulence of Pantoea ananatis in onion seedlings. Mol Plant Pathol. 2016;18:734-45.

48. Braun PG, Hildebrand PD. Infection, carbohydrate utilization, and protein profiles of apple, pear, and raspberry isolates of Erwinia amylovora. Can J Plant Pathol. 2005:27:338-46.

49. Zhang ZF, Nan ZB. Erwinia persicina, a possible new necrosis and wilt threat to forage or grain legumes production. Eur J Plant Pathol. 2014;139:349-58.

50. Zimmermann $\mathrm{MH}$, Ziegler $\mathrm{H}$. List of sugars and sugar alcohols in sieve-tube exudates. In: Zimmermann MH, Milburn JA, editors. Encyclopedia of plant physiology. New York: Springer-Verlag; 1975. p. 480-503.

51. Hardoim PR, Nazir R, Sessitsch A, Elhottova D, Korenblum E, van Overbeek LS, et al. The new species Enterobacter oryziphilus sp. nov. and Enterobacter oryzendophyticu sp. nov. are key inhabitants of the endosphere of rice. BMC Microbiol. 2013;13:1-13.

52. Waleron M, Waleron K, Lojkowska E. Characterization of Pectobacterium carotovorum subsp. odoriferum causing soft rot of stored vegetables. Eur J Plant Pathol. 2014;139:457-69.

53. Waleron M, Waleron K, Lojkowska E. First report of Pectobacterium carotovorum subsp. brasiliense causing soft rot on potato and other vegetables in Poland. Plant Dis. 2015;99:1271.

54. Perombelon MCM, van der Wolf JM. Methods for the detection and quantification of Erwinia carotovora on potatoes: a laboratory manual. Dundee: Scottish Crop Research Institute Occasional Publication; 2002.

55. Sareh BR, Heshmat R, Masoud SB. Characterization of Pectobacterium species from Iran using biochemical and molecular methods. Eur J Plant Pathol. 2011;129:413-25.

56. Gardan L, Gouy C, Christen R. Elevation of three subspecies of Pectobacterium carotovorum to species level: Pectobacterium atrosepticum sp. nov., Pectobacterium betavasculorum sp. nov. and Pectobacterium wasabiae sp. nov. Int J Syst Evol Micr. 2003;53:381-91.

57. Oskiera M, KałużNa M, Kowalska B, SmoliniSka U. Pectobacterium carotovorum subsp. odoriferum on cabbage and Chinese cabbage: identification, characterization and taxonomic relatedness of bacterial soft rot causal agents. J Plant Pathol. 2017;99:149-60.

58. Duarte V, de Boer SH, Ward LJ, de Oliveira AM. Characterization of atypical Erwinia carotovora strains causing blackleg of potato in Brazil. J Appl Microbiol. 2004;96:535-45.

59. Schaad NW, Jones JB, Chun W. Laboratory guide for identification of plant pathogenic Bacteria. 3rd ed. USA: American Phytopathological Society Press; 2001.

60. Chojniak J, Jałowiecki Ł, Dorgeloh E, Hegedusova B, Ejhed H, Magnér J, et al. Application of the BIOLOG system for characterization of Serratia marcescens ss marcescens isolated from onsite wastewater technology (OSWT)*. Acta Biochim Pol. 2015;62:799-805.

61. Lowe TM, Eddy SR. tRNAscan-SE: a program for improved detection of transfer RNA genes in genomic sequence. Nucleic Acids Res. 1997;25:955-64.

62. Lagesen K, Hallin P, Rodland E, Staerfeldt H, Rognes T, Ussery D. RNAmmer: consistent and rapid annotation of ribosomal RNA genes. Nucleic Acids Res. 2007;35:3100-8. 
63. Zhou CE, Smith J, Lam M, Zemla A, Dyer MD, Slezak T. Mvirdb-a microbial database of protein toxins, virulence factors and antibiotic resistance genes for bio-defence applications. Nucleic Acids Res. 2007;35:391-4.

64. Kent WJ. BLAT-the BLAST-like alignment tool. Genome Res. 2002;12:656-64.

65. Muhire B, Martin DP, Brown JK, Navas-Castillo J, Moriones E, Zerbini FM, et al. A genome-wide pairwise-identity-based proposal for the classification of viruses in the genus Mastrevirus (family Geminiviridae). Arch Virol. 2013;158: $1411-24$.

66. Li W, Godzik A. Cd-hit: a fast program for clustering and comparing large sets of protein or nucleotide sequences. Bioinformatics. 2006;22:1658-9.

67. Huang Y, Niu B, Gao Y, Fu L, Li W. CD-HIT suite: a web server for clustering and comparing biological sequences. Bioinformatics. 2010;26:680-2.

68. Makarova KS, Haft DH, Barrangou R, Brouns SJJ, Charpentier E, Horvath P, et al. Evolution and classification of the CRISPR-Cas systems. Nat Rev Microbiol. 2011;9:467-77.

Ready to submit your research? Choose BMC and benefit from:

- fast, convenient online submission

- thorough peer review by experienced researchers in your field

- rapid publication on acceptance

- support for research data, including large and complex data types

- gold Open Access which fosters wider collaboration and increased citations

- maximum visibility for your research: over $100 \mathrm{M}$ website views per year

At $\mathrm{BMC}$, research is always in progress.

Learn more biomedcentral.com/submissions 